\title{
Effects of mechanical feedback on the stability of cardiac scroll waves: A bidomain electro-mechanical simulation study
}

P. Colli Franzone, L. F. Pavarino, and S. Scacchi

Citation: Chaos 27, 093905 (2017); doi: 10.1063/1.4999465

View online: https://doi.org/10.1063/1.4999465

View Table of Contents: http://aip.scitation.org/toc/cha/27/9

Published by the American Institute of Physics

\section{Articles you may be interested in}

Introduction to Focus Issue: Complex Cardiac Dynamics

Chaos: An Interdisciplinary Journal of Nonlinear Science 27, 093701 (2017); 10.1063/1.5003940

Modeling dynamics in diseased cardiac tissue: Impact of model choice

Chaos: An Interdisciplinary Journal of Nonlinear Science 27, 093909 (2017); 10.1063/1.4999605

Slow $\left[\mathrm{Na}^{+}\right]_{i}$ dynamics impacts arrhythmogenesis and spiral wave reentry in cardiac myocyte ionic model

Chaos: An Interdisciplinary Journal of Nonlinear Science 27, 093907 (2017); 10.1063/1.4999475

Assessing multiscale complexity of short heart rate variability series through a model-based linear approach

Chaos: An Interdisciplinary Journal of Nonlinear Science 27, 093901 (2017); 10.1063/1.4999353

Distinguishing mechanisms for alternans in cardiac cells using constant-diastolic-interval pacing

Chaos: An Interdisciplinary Journal of Nonlinear Science 27, 093902 (2017); 10.1063/1.4999354

Measurement and structure of spiral wave response functions

Chaos: An Interdisciplinary Journal of Nonlinear Science 27, 093912 (2017); 10.1063/1.4999606

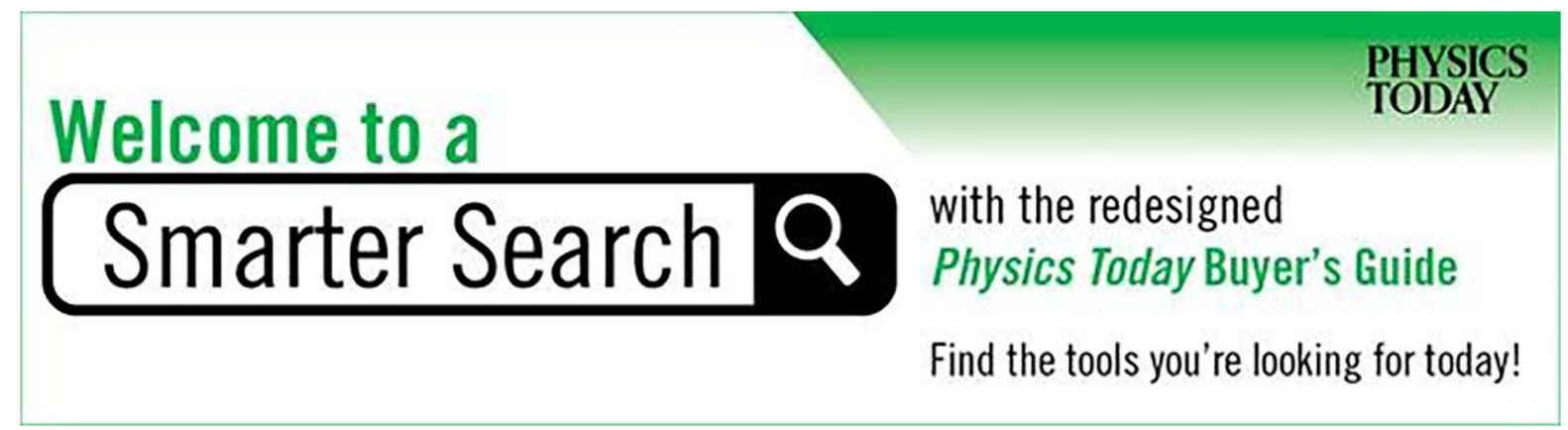




\title{
Effects of mechanical feedback on the stability of cardiac scroll waves: A bidomain electro-mechanical simulation study
}

\author{
P. Colli Franzone, ${ }^{1, a)}$ L. F. Pavarino, ${ }^{1, b)}$ and S. Scacchi ${ }^{2, c)}$ \\ ${ }^{1}$ Dipartimento di Matematica, Università di Pavia, Via Ferrata 1, 27100 Pavia, Italy \\ ${ }^{2}$ Dipartimento di Matematica, Università di Milano, Via Saldini 50, 20133 Milano, Italy
}

(Received 20 March 2017; accepted 20 June 2017; published online 23 August 2017)

\begin{abstract}
In this work, we investigate the influence of cardiac tissue deformation on re-entrant wave dynamics. We have developed a 3D strongly coupled electro-mechanical Bidomain model posed on an ideal monoventricular geometry, including fiber direction anisotropy and stretch-activated currents (SACs). The cardiac mechanical deformation influences the bioelectrical activity with two main mechanical feedback: (a) the geometric feedback (GEF) due to the presence of the deformation gradient in the diffusion coefficients and in a convective term depending on the deformation rate and (b) the mechano-electric feedback (MEF) due to SACs. Here, we investigate the relative contribution of these two factors with respect to scroll wave stability. We extend the previous works [Keldermann et al., Am. J. Physiol. Heart Circ. Physiol. 299, H134-H143 (2010) and Hu et al., PLoS One 8(4), e60287 (2013)] that were based on the Monodomain model and a simple non-selective linear SAC, while here we consider the full Bidomain model and both selective and non-selective components of SACs. Our simulation results show that the stability of cardiac scroll waves is influenced by MEF, which in case of low reversal potential of non-selective SACs might be responsible for the onset of ventricular fibrillation; GEF increases the scroll wave meandering but does not determine the scroll wave stability. Published by AIP Publishing. [http://dx.doi.org/10.1063/1.4999465]
\end{abstract}

Ventricular fibrillation is a life-threatening cardiac arrhythmia consisting of a turbulent and disorganized electrical activity of the heart, due to the breakup of reentrant excitation sources called scroll waves. Understanding the mechanisms which lead to the onset of ventricular fibrillation is one of the most important issues in electrocardiology. In the last two decades, several simulations studies have addressed this issue, showing that one of the possible causes of scroll waves breakup and fibrillation is a steep action potential duration (APD) restitution curve. Recent works based on cardiac electro-mechanical simulations have investigated the role of mechano-electric feedback (MEF) induced by stretch activated currents (SAC) in determining and maintaining ventricular fibrillation. The aim of the present paper is to study the influence of mechanical deformation on the generation and stability of cardiac scroll waves by means of three-dimensional Bidomain electro-mechanical simulations. Our electromechanical coupling model takes into account the two main mechanical feedback on the bioelectrical activity: the geometric feedback (GEF) due to the presence of the deformation gradient in the diffusion coefficients and in a convective term depending on the deformation rate, and the mechano-electric feedback (MEF) due to the presence of stretch-activated currents through the myocyte membrane. Our simulations results show that the stability of cardiac scroll waves is influenced by MEF, which in some cases might be responsible for their breakup, leading to the onset of ventricular fibrillation.

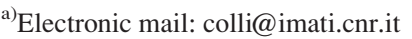

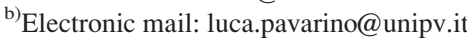

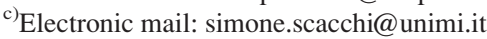

\section{INTRODUCTION}

Abnormal electrical activity of the heart may result in dangerous cardiac arrhythmias, such as ventricular tachycardia. In some patients, ventricular tachycardia leads to ventricular fibrillation, a situation where ventricles twitch randomly instead of contracting in a co-ordinated way and that is the most common cause of death in the industrialized countries. Both clinical and experimental studies have shown that ventricular fibrillation consists of a turbulent and disorganized electrical activity of the heart, due to the breakup of reentrant excitation sources called scroll waves, see e.g., Refs. 20, 31, and 47. The mechanisms underlying the onset of ventricular fibrillation are of great interest and still a matter of debate in the cardiology community. Previous simulation studies have investigated the multiple mechanisms of spiral and scroll wave breakup, see, e.g., Refs. 6, 16, 17, 56, 57, 65, 71, and 76 showing, in particular, that steep action potential duration (APD) restitution curves play a role in determining scroll waves breakup.

The aim of the current study is to investigate the influence of myocardial deformation on the maintenance of ventricular tachycardia induced by premature beats, and on its degeneration to ventricular fibrillation. Cardiac mechanical deformation influences the bioelectrical activity with two main mechanical feedback: (a) the geometric feedback (GEF) due to the presence of the deformation gradient in the diffusion coefficients and in a convective term depending on the deformation rate, and (b) the mechano-electric feedback (MEF) due to the presence of stretch-activated channels in the myocyte membrane.

Computer modeling represents today a very useful tool for investigating the effect of mechanical contraction on the 
cardiac bioelectrical activity, since the recording of simultaneous electrical and mechanical experimental data with high spatial and temporal resolution is not yet available. Currently, most techniques of optical transmembrane potential mapping use electro-mechanical uncoupling agents to abolish heart motion; see Ref. 27 for a review. Recent studies ${ }^{43,59,62,73}$ have used motion tracking techniques to map the regional deformation of a contracting heart. These techniques hold promise for recording the transmembrane optical mapping in beating heart preparations, thus enabling the experimental study of electromechanical coupling. Alternatively, in order to predict the optical action potential recorded from the beating myocardial surface, the forward problem could be solved by combining realistic electro-mechanical models with a photon-diffusion model (taking into account the transport and scattering during the process of illumination and emission); these techniques have been developed in the past using only electrophysiological bidomain models (without mechanics) in the Refs. 4, 5, and 25.

In recent years, several cardiac electro-mechanical models have been developed in order to describe the coupling between the bioelectrical activity and the mechanical deformation of the cardiac tissue, see, e.g., Refs. 2, 12, 13, 15, 18, $19,23,24,37,42,48,52,60,68$, and 75. In particular, initial studies on the effect of mechano-electric feedback via stretch-activated current on cardiac bioelectrical activity and ventricular reentrant wave induction and stability can be found in Refs. 1, 26, 29, 33, 35, 44, and 70.

In our previous works, ${ }^{9-11}$ we have developed a threedimensional strongly coupled electro-mechanical model consisting of four sub-models. The first two, describing the electrical and mechanical properties of the cardiac tissue at macroscopic level, are the Bidomain model, see, e.g., Ref. 8 , posed in a deformable tissue domain with transversely isotropic conductivity; the quasi-static finite elasticity equations, based on a transversely isotropic strain energy function derived from that proposed in Ref. 14. The other two describe the electrical and mechanical interactions at the microscopic level of the myocyte and are the ten Tusscher ${ }^{63,64}$ ionic membrane model for human ventricular myocytes; the mechanistic active tension model proposed in Ref. 41.

In this work, we investigate the relative contribution of the geometrical mechano-electric feedback and the stretchactivated currents to scroll wave stability. We extend the findings of two early studies by Keldermann et al. ${ }^{35}$ and $\mathrm{Hu}$ et al. $^{29}$ that were based on the simpler Monodomain model or a simple non-selective linear stretch-activated current, while here we consider the full Bidomain model and we incorporate two different stretch-activated currents, i.e., the non-selective and the potassium selective components varying the degree of their expression.

\section{METHODS}

\section{A. The cardiac electro-mechanical coupling model}

We start with a brief description of the four mechanical and bioelectrical components of our strongly coupled electromechanical model.

\section{Mechanical model of cardiac tissue}

We denote by $\mathbf{X}=\left(X_{1}, X_{2}, X_{3}\right)^{T}$ the material coordinates of the undeformed cardiac domain $\hat{\Omega}$, by $\mathbf{x}=\mathbf{x}(\mathbf{X}, t)$ the spatial coordinates of the deformed cardiac domain $\Omega(t)$ at time $t$, by $\mathbf{F}(\mathbf{X}, t)=\frac{\partial \mathbf{x}}{\partial \mathbf{X}}$ the deformation gradient and by $\mathbf{u}(\mathbf{X}, t)$ $=\mathbf{x}-\mathbf{X}$ the displacement field.

Assuming a quasi-steady state regime, the cardiac tissue is modeled as a non-linear hyperelastic material satisfying the force equilibrium equation

$$
\operatorname{Div}(\mathbf{F S})=0, \quad \mathbf{X} \in \hat{\Omega},
$$

with appropriate boundary conditions described below. Because the active stress approach is assumed, the second Piola-Kirchhoff stress tensor $\mathbf{S}$ is given by the sum of passive (pas), volumetric ( $v o l)$, and active (act) components, i.e.,

$$
\mathbf{S}=\mathbf{S}^{p a s}+\mathbf{S}^{v o l}+\mathbf{S}^{a c t} .
$$

The passive and volumetric components are defined as

$$
S_{i j}^{p a s, v o l}=\frac{1}{2}\left(\frac{\partial W^{p a s, v o l}}{\partial E_{i j}}+\frac{\partial W^{p a s, v o l}}{\partial E_{j i}}\right) \quad i, j=1,2,3,
$$

where $\mathbf{E}=\frac{1}{2}(\mathbf{C}-\mathbf{I})$ is the Green-Lagrange strain tensor and $W^{p a s}$ is an exponential strain energy function modeling the myocardium as a transversely isotropic hyperelastic material (derived from the orthotropic law proposed in Refs. 14 and 28), $W^{v o l}=K(J-1)^{2}$ is a volume change penalization term added in order to model the myocardium as nearly incompressible, with $K$ a positive bulk modulus and $J=\operatorname{det} \mathbf{F}$.

\section{Mechanical model of active tension}

The contraction of ventricles results from the active tension generated by the model of myofilaments dynamics activated by calcium. We assume that the generated active force acts only along the fiber direction; hence, the active Cauchy stress is

$$
\sigma^{a c t}(\mathbf{x}, t)=T_{a} \mathbf{a}_{l}(\mathbf{x}) \otimes \mathbf{a}_{l}(\mathbf{x}),
$$

where $\mathbf{a}_{l}$ is a unit vector parallel to the local fiber direction and $T_{a}$ is the active fiber stress related to the deformed domain. The unit vector parallel to the local fiber in the deformed configuration can be written as

$$
\mathbf{a}_{l}=\frac{\mathbf{F} \hat{\mathbf{a}}_{l}}{\left\|\mathbf{F} \hat{\mathbf{a}}_{l}\right\|}=\frac{\mathbf{F} \hat{\mathbf{a}}_{l}}{\sqrt{\hat{\mathbf{a}}_{l}^{T} \mathbf{C} \hat{\mathbf{a}}_{l}}},
$$

where $\hat{\mathbf{a}}_{l}$ is the fiber direction in the reference configuration. In terms of the principal axes of the reference configuration, we obtain

$$
\mathbf{a}_{l} \otimes \mathbf{a}_{l}=\frac{\mathbf{F} \hat{\mathbf{a}}_{l} \otimes \mathbf{F} \hat{\mathbf{a}}_{l}}{\left\|\mathbf{F} \hat{\mathbf{a}}_{l}\right\|^{2}}=\frac{\mathbf{F} \hat{\mathbf{a}}_{l} \hat{\mathbf{a}}_{l}^{T} \mathbf{F}^{T}}{\hat{\mathbf{a}}_{l}^{T} \mathbf{C} \hat{\mathbf{a}}_{l}} .
$$

Then, the second Piola-Kirchhoff active stress component is given by 


$$
\mathbf{S}^{a c t}=J \mathbf{F}^{-1} \sigma^{a c t} \mathbf{F}^{-T}=J T_{a} \frac{\hat{\mathbf{a}}_{l} \otimes \hat{\mathbf{a}}_{l}}{\hat{\mathbf{a}}_{l}^{T} \mathbf{C} \hat{\mathbf{a}}_{l}} .
$$

The biochemically generated active tension

$$
T_{a}=T_{a}\left(C a_{i}, \lambda, \frac{d \lambda}{d t}\right)
$$

depends on the intracellular calcium concentration $C a_{i}$, the fiber stretch $\lambda=\sqrt{\hat{\mathbf{a}}_{l}^{T} \mathbf{C} \hat{\mathbf{a}}_{l}}$, and the stretch-rate $\frac{d \lambda}{d t}$ along the fiber direction.

The active tension generation model was based on a calcium kinetic and myofilament dynamics, see, e.g., Refs. 32, 49,58 , and 61 . In this work, we consider the framework proposed in Ref. 32 updated in Ref. 49 and recently modified in Land et $a .^{41}$

\section{Bioelectrical model of cardiac tissue: The Bidomain model}

In Ref. 10, we have shown how, starting from the charge conservation law on the deformed configuration, imposing the quasi-static regime and disregarding ionic diffusion with respect to drift currents, the Bidomain model, usually written on a fixed domain, can be extended to a moving domain. We denote by $v, u_{e}, \mathbf{w}$, and $\mathbf{c}$ the transmembrane potential, the extracellular potential, the gating and ionic concentrations variables on the deformed configuration and by $\hat{v}, \hat{u}_{e}, \hat{\mathbf{w}}, \hat{\mathbf{c}}$ the same quantities on the reference configuration. Then, the parabolic-elliptic formulation of the Bidomain model written on the deformed configuration $\Omega(t)$ is given by

$$
\left\{\begin{array}{l}
c_{m} \frac{\partial v}{\partial t}-\operatorname{div}\left(D_{i} \nabla\left(v+u_{e}\right)\right)+i_{i o n}(v, \mathbf{w}, \mathbf{c}, \lambda)=i_{a p p}^{i} \\
-\operatorname{div}\left(D_{i} \nabla v\right)-\operatorname{div}\left(\left(D_{i}+D_{e}\right) \nabla u_{e}\right)=i_{a p p}^{i}+i_{a p p}^{e}
\end{array}\right.
$$

where $c_{m}$ and $i_{\text {ion }}$ are the membrane capacitance and ionic current per unit volume, respectively, and in order to satisfy the compatibility condition $\int_{\Omega(t)}\left(i_{a p p}^{i}+i_{a p p}^{e}\right) d \mathbf{x}=0$, we choose $i_{a p p}^{i}=-i_{a p p}^{e}=i_{a p p}$. In the Lagrangian framework, after the pull-back on the reference configuration $\hat{\Omega} \times(0, T)$, this system becomes

$$
\left\{\begin{array}{l}
c_{m} J\left(\frac{\partial \hat{v}}{\partial t}-\mathbf{F}^{-T} \operatorname{Grad} \hat{v} \cdot \mathbf{V}\right) \\
\quad-\operatorname{Div}\left(J \mathbf{F}^{-1} \hat{D}_{i} \mathbf{F}^{-T} \operatorname{Grad}\left(\hat{v}+\hat{u}_{e}\right)\right) \\
+J i_{i o n}(\hat{v}, \hat{\mathbf{w}}, \hat{\mathbf{c}}, \lambda)=J \hat{i}_{a p p}, \\
\quad-\operatorname{Div}\left(J \mathbf{F}^{-1} \hat{D}_{i} \mathbf{F}^{-T} \operatorname{Grad} \hat{v}\right) \\
\quad-\operatorname{Div}\left(J \mathbf{F}^{-1}\left(\hat{D}_{i}+\hat{D}_{e}\right) \mathbf{F}^{-T} \operatorname{Grad} \hat{u}_{e}\right)=0
\end{array}\right.
$$

where $\mathbf{V}=\frac{\partial \mathbf{u}}{\partial t}$ is the rate of deformation; see Ref. 10 for the detailed derivation. These two partial differential equations (PDEs) are coupled through the reaction term $i_{\text {ion }}$ with the ordinary differential equation (ODE) system of the membrane model, given in $\Omega(t) \times(0, T)$ by

$$
\frac{\partial \mathbf{w}}{\partial t}-\mathbf{R}_{w}(v, \mathbf{w})=0, \quad \frac{\partial \mathbf{c}}{\partial t}-\mathbf{R}_{c}(v, \mathbf{w}, \mathbf{c})=0 .
$$

The bioelectrical system [(5) and (6)] is completed by prescribing initial conditions on $\hat{v}, \mathbf{w}, \mathbf{c}$, insulating boundary conditions on $\hat{u}_{e}, \hat{u}_{i}=\hat{v}+\hat{u}_{e}$, and the intra- and extracellular applied current $\hat{i}_{\text {app }}=\hat{i}_{\text {app }}=-\hat{i}_{\text {app }}$. We recall that the extracellular potential $\hat{u}_{e}$ is defined up to a time dependent constant in space $R(t)$ determined by the choice of the reference potential. In this paper, we consider as a reference potential the average of the extracellular potential over the cardiac volume, i.e., we impose $\int_{\hat{\Omega}} \hat{u}_{e}(\mathbf{X}, t) J(\mathbf{X}, t) d \mathbf{X}=0$. Assuming transversely isotropic properties of the intra- and extracellular media, the conductivity tensors on the deformed configuration are given by

$$
D_{i, e}=\sigma_{t}^{i, e} I+\left(\sigma_{l}^{i, e}-\sigma_{t}^{i, e}\right) \mathbf{a}_{l} \otimes \mathbf{a}_{l},
$$

where $\sigma_{l}^{i, e}, \sigma_{t}^{i, e}$ are the conductivity coefficients of the intraand extracellular media measured along the fiber direction $\mathbf{a}_{l}$ and any cross fiber direction, respectively. From (3), it follows that the tensors $D_{i, e}(x, t)$ written on the reference configuration are

$$
\begin{aligned}
\hat{D}_{i, e}(\mathbf{X}, t) & =D_{i, e}(\mathbf{x}(\mathbf{X}, t), t)= \\
& =\sigma_{t}^{i, e} I+\left(\sigma_{l}^{i, e}-\sigma_{t}^{i, e}\right) \frac{\mathbf{F} \hat{\mathbf{a}}_{l} \hat{\mathbf{a}}_{l}^{T} \mathbf{F}^{T}}{\hat{\mathbf{a}}_{l}^{T} \mathbf{C} \hat{\mathbf{a}}_{l}^{T}} .
\end{aligned}
$$

Therefore, the equivalent conductivity tensors appearing into the bidomain model written in the reference configuration are given by

$$
J \mathbf{F}^{-1} \hat{D}_{i, e}(\mathbf{X}, t) \mathbf{F}^{-T}=\sigma_{t}^{i, e} \mathbf{C}^{-1}+\left(\sigma_{l}^{i, e}-\sigma_{t}^{i, e}\right) \frac{\hat{\mathbf{a}}_{l} \hat{\mathbf{a}}_{l}^{T}}{\hat{\mathbf{a}}_{l}^{T} \mathbf{C} \hat{\mathbf{a}}_{l}^{T}} .
$$

\section{Ionic membrane model and stretch-activated channel current}

The ionic current in the Bidomain model (5) is given by $i_{\text {ion }}=\chi I_{\text {ion }}$, where $\chi$ is the membrane surface to volume ratio and the ionic current per unit area of the membrane surface $I_{i o n}$ is given by the sum $I_{i o n}(v, \mathbf{w}, \mathbf{c}, \lambda)=I_{i o n}^{m}(v, \mathbf{w}, \mathbf{c})+I_{s a c}$ of the ionic term $I_{i o n}^{m}(v, \mathbf{w}, \mathbf{c})$ given by the ten Tusscher model (TP06), ${ }^{63,64}$ available from the cellML depository (models.cellml.org/cellml), and a stretch-activated current $I_{s a c}$. The TP06 ionic model also specifies the functions $R_{w}(v$, $\mathbf{w})$ and $R_{c}(v, \mathbf{w}, \mathbf{c})$ in the ODE system (6), consisting of 17 ordinary differential equations modeling the main ionic currents dynamics.

Experimental investigation and modeling of stretch activated currents (SAC) are presented in Refs. 26, 30, 34, 39, 40, 45, 50, 70, 72, and 74, see also Refs. 38 and 55. Experimental data support an almost linear dependence of stretch-activated currents on the fiber stretch when $\lambda \geq 1$. These works have shown that the effects of stretch on transmembrane potential depends on stretch timing and magnitude, and on the reversal potential of the two main components of the stretch-activated current 


$$
I_{s a c}=I_{n s}+I_{K o}
$$

i.e., the non-selective component $I_{n s}$ and the potassium selective component $I_{K o}$. In particular, during the resting phase, $I_{K o}$, with a reversal potential lower than the resting one, tends to hyperpolarize the cell, whereas $I_{n s}$, with a reversal potential higher than the resting one, depolarizes the cell. During the action potential, $I_{K o}$ continues to produce a hyperpolarizing effect, facilitating repolarization, while $I_{n s}$ has a hyperpolarizing effect only when the transmembrane potential is higher than its reversal potential. Therefore, $I_{n s}$ shortens APD during early repolarization, but may induce APD prolongation or even after-depolarization events during late repolarization.

In this work, we adopt the model of $I_{s a c}=I_{n s}+I_{K o}$ proposed in Ref. 50 where the non-selective current is defined by

$$
I_{n s}=I_{n s, N a}+I_{n s, K}=g_{n s} \gamma_{s l}(\lambda)\left[r\left(v-v_{N a}\right)+\left(v-v_{K}\right)\right],
$$

with $\gamma_{s l}(\lambda)=10 \max (\lambda-1,0), g_{n s}=4.13 \times 10^{-3} \mathrm{mS} / \mathrm{cm}^{2}$ and the value of $r$ measures the relative conductance of $\mathrm{Na}^{+}$ with respect to $K^{+}$. The current relation can be rewritten as

$$
I_{n s}=(r+1) g_{n s} \gamma_{s l}(\lambda)\left(v-v_{n s}\right), \quad \text { with } v_{n s}=\frac{r v_{n a}+v_{k}}{r+1} .
$$

The value of $r$, as well as measuring the relative conductance of the ions $\mathrm{Na}^{+}$and $\mathrm{K}^{+}$, determines the reversal potential $v_{n s}$ of $I_{n s}$ varying the degree of expression of the ions $\mathrm{Na}^{+}$ and $K^{+}$.

The $K^{+}$selective SAC current is defined by

$$
I_{K o}=g_{K o} \frac{\gamma_{S L, K o}}{1+\exp (-(10+v) / 45)}\left(v-v_{K}\right),
$$

where $g_{K o}=1.2 \times 10^{-2} \mathrm{mS} / \mathrm{cm}^{2}$ and $\gamma_{S L, K o}=3 \max (\lambda-1,0)$ +0.7 .

In the simulations presented in Sec. III, we consider two different types of $I_{n s}$ :

- with a reversal potential $v_{n s}=-60 \mathrm{mV}$, corresponding to $r$ $=0.2$, which represents a higher degree of expression of $K^{+} I_{s a c}$, resulting in APD shortening depending on its timing and amplitude;

- with a reversal potential $v_{n s}=-10 \mathrm{mV}$, corresponding to $r$ $=1$, which represents a higher degree of expression of $\mathrm{Na}^{+} I_{\text {sac }}$, resulting in APD prolongation depending on its timing and amplitude.

Indeed, for $r=1$, the value of conductance is double and since the reversal potential for $I_{n s}$ is $v_{n s}=-10 \mathrm{mV}$, this corresponds to a prevailing role of $\mathrm{Na}^{+}$in the non-selective

Panel A: $I_{s a c}$ calibration, $\lambda=1.3$ and $r=0.2$

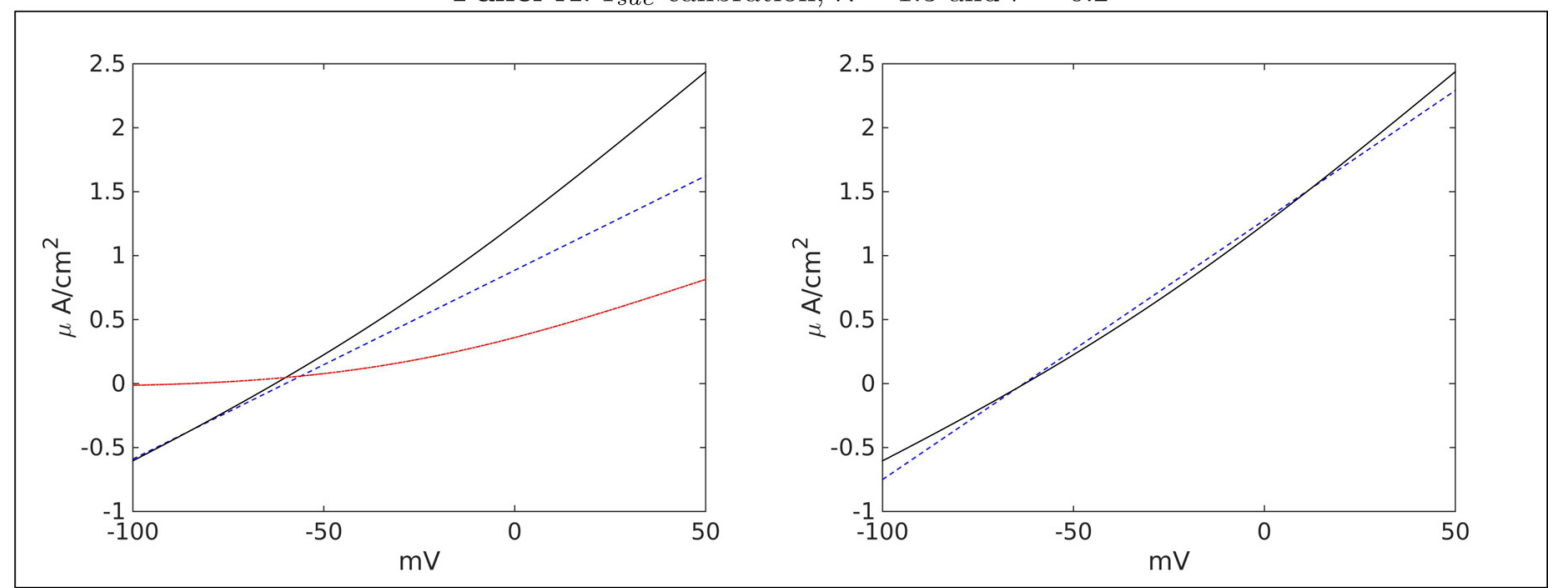

Panel B: $I_{s a c}$ calibration, $\lambda=1.3$ and $r=1$

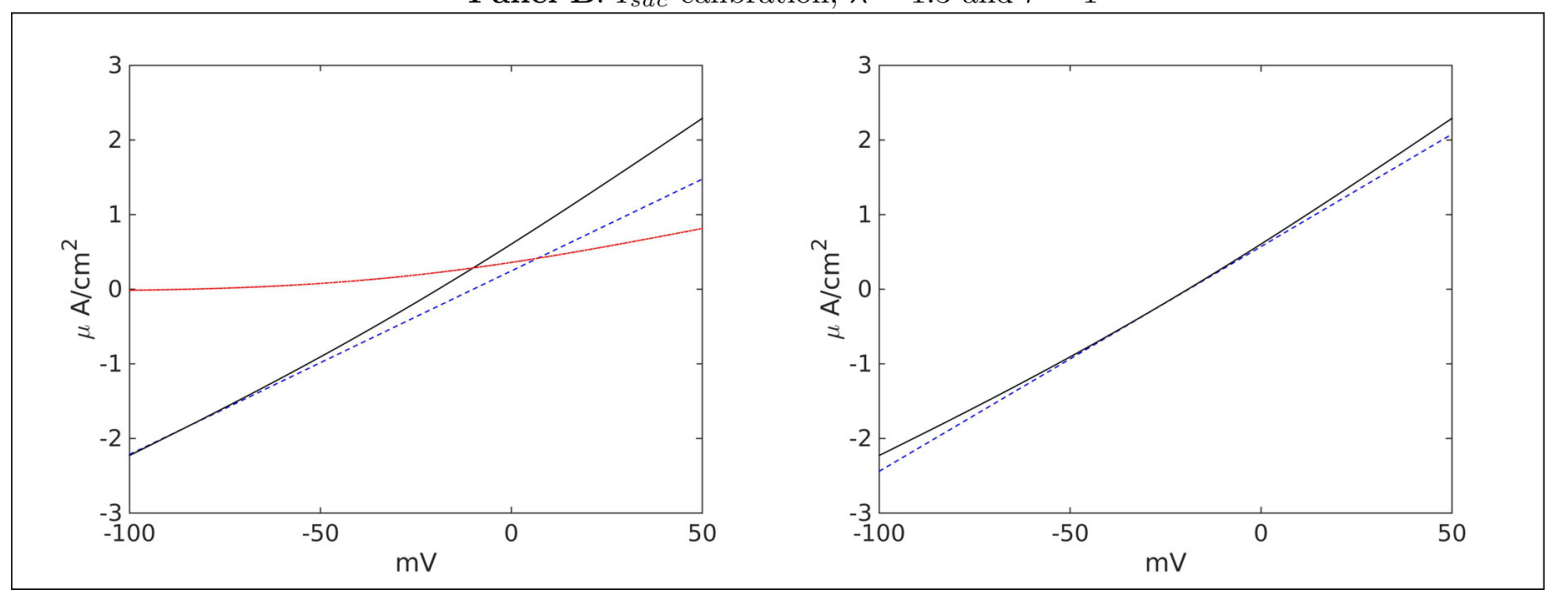

FIG. 1. Panel A: $I_{s a c}$ calibration, $\lambda=1.3$ and $r=0.2$. Left: plot of $I_{n s}(v)$ (blue dashed), $I_{K o}(v)$ (red), and $I_{s a c}(v)$ (black). Right: plot of $I_{s a c}$ (black) and $I_{s}$ (blue dashed). Panel B: $I_{\text {sac }}$ calibration, $\lambda=1.3$, and $r=1$. Same format as in panel A. 
current, which in the systolic phase yields a repolarizing current resulting in APD prolongation of the myocyte. For $r=0.2$, the value of conductance is reduced by one fifth and since the reversal potential for $I_{n s}$ is $v_{n s}=-60 \mathrm{mV}$, this yields a higher degree of expression of $K^{+}$in the non-selective current $I_{n s}$, which in the systolic phase generates a depolarizing current inducing an APD shortening of the myocyte.

The total $I_{s a c}$ current exhibits an almost linear dependence on the transmembrane potential $v$ as shown in Fig. 1 (panel A-left and panel B-left). Moreover, for the previous two settings, the total $I_{s a c}$ exhibits a reversal potential slightly modified with respect to the isolated $I_{n s}$ component, amounting to about -19.1 and $-62.7 \mathrm{mV}$ for $r=1$ and $r=0.2$, respectively.

The total $I_{s a c}$ model, composed by non-selective and $K^{+}$ selective channels, for the two reversal parameter settings can be approximated by a linear relation

$$
I_{s}(v)=g_{s} \gamma_{s}(\lambda)\left(v-v_{s a c}\right),
$$

where $\gamma_{s}(\lambda)=\beta_{s} \max (\lambda-1,0)$, with strain coefficient $\beta_{s}$ $=10$. This linear approximation is confirmed by the plots in Fig. 1 (panel A-right and panel B-right). More precisely, the approximated linear-relation is given by

$$
\begin{aligned}
& \text { for } \mathrm{r}=1: v_{s} \approx-19.1 \mathrm{mV} g_{s} \approx 10^{-2} \mathrm{mS} / \mathrm{cm}^{2} \\
& \text { for } \mathrm{r}=0.2: v_{s} \approx-62.7 \mathrm{mV} g_{s} \approx 0.68 \times 10^{-2} \mathrm{mS} / \mathrm{cm}^{2} .
\end{aligned}
$$

These values are in the range $10^{-3} \leftrightarrow 5 \times 10^{-2} \mathrm{mS} / \mathrm{cm}^{2}$, which includes the conductance values used in most computational studies employing a linear model of $I_{\text {sac }}$, see, e.g., Refs. 29, 44, 46, and 66.

\section{B. Numerical methods, parameters calibration, and simulations setup}

\section{Domain geometry}

The cardiac domain $\hat{\Omega}=\Omega(0)$ is the image of a Cartesian periodic slab using ellipsoidal coordinates, yielding a truncated ellipsoid modeling a left ventricular geometry, described by the parametric equations

$$
\begin{cases}x=a(r) \cos \theta \cos \phi & \phi_{\text {min }} \leq \phi \leq \phi_{\text {max }} \\ y=b(r) \cos \theta \sin \phi & \theta_{\text {min }} \leq \theta \leq \theta_{\text {max }} \\ z=c(r) \sin \theta & 0 \leq r \leq 1\end{cases}
$$

where $a(r)=a_{1}+r\left(a_{2}-a_{1}\right), b(r)=b_{1}+r\left(b_{2}-b_{1}\right), c(r)$ $=c_{1}+r\left(c_{2}-c_{1}\right), \quad$ and $\quad a_{1}=b_{1}=1.5, a_{2}=b_{2}=2.7, c_{1}$ $=4.4, c_{2}=5$ (all in $\mathrm{cm}$ ) and $\phi_{\min }=-\pi / 2, \phi_{\max }=3 \pi / 2$, $\theta_{\text {min }}=-3 \pi / 8, \theta_{\max }=\pi / 8$. We will refer to the inner surface of the truncated ellipsoid $(r=0)$ as endocardium and to the outer surface $(r=1)$ as epicardium. The fibers rotate intramurally linearly with the depth for a total amount of $120^{\circ}$ proceeding counterclockwise from epicardium to endocardium. More precisely, in a local ellipsoidal reference system $\left(\mathbf{e}_{\phi}, \mathbf{e}_{\theta}, \mathbf{e}_{r}\right)$, the fiber direction $\mathbf{a}_{l}(\mathbf{x})$ at a point $\mathbf{x}$ is given by $\mathbf{a}_{l}(\mathbf{x})=\mathbf{b}_{l}(\mathbf{x}) \cos (\beta)+\mathbf{n}(\mathbf{x}) \cos (\beta)$, where

$$
\mathbf{b}_{l}(\mathbf{x})=\mathbf{e}_{\phi} \cos \alpha(r)+\mathbf{e}_{\theta} \sin \alpha(r),
$$

with

$$
\alpha(r)=\frac{2}{3} \pi(1-r)-\frac{\pi}{4}, \quad 0 \leq r \leq 1,
$$

where $\mathbf{n}(\mathbf{x})$ is the unit outward normal to the ellipsoidal surface at $\mathbf{x}$ and $\beta$ is the imbrication angle calibrated as in Ref. 7 given by $\beta=\arctan (\cos \alpha \tan \gamma)$, with $\gamma=\theta(1-r) 60 / \pi$.

\section{Space discretization}

We discretize the cardiac domain with a hexahedral structured grid $T_{h_{m}}$ for the mechanical model (1) and $T_{h_{e}}$ for the Bidomain model (5), where $T_{h_{e}}$ is a refinement of $T_{h_{m}}$, i.e., $h_{m}$ is an integer multiple of $h_{e}$. We consider the variational formulations of both mechanical and bioelectrical models and then approximate all scalar and vector fields by isoparametric $Q_{1}$ finite elements in space. In all the electromechanical simulations, we employ an electrical mesh size $h_{e}=0.01 \mathrm{~cm}$ in order to properly resolve the sharp excitation front, while the smoother mechanical deformation allow us to use a coarse mechanical mesh of size $h_{m}=0.08 \mathrm{~cm}$. The resulting electrical mesh consists of $N_{\phi} \times N_{\theta} \times N_{k}=384 \times 192 \times 48$ elements, yielding about $3.63 \times 10^{6}$ nodes, while the mechanical mesh consists of $48 \times 24 \times 6$ elements, yielding about 8400 nodes.

\section{Time discretization}

The time discretization of the electromechanical model is performed by the following semi-implicit splitting method, where the electrical and mechanical time steps could be different. At each time step,

(a) given $v^{n}, w^{n}, c^{n}$ at time $t_{n}$, solve the ODE system of the membrane model (6) with a first order implicit-explicit (IMEX) method to compute the new $w^{n+1}, c^{n+1}$;

(b) given the calcium concentration $C a_{i}^{n+1}$, which is included in the concentration variables $c^{n+1}$, solve the variational formulation of the mechanical problem (1) and the active tension system to compute the new deformed coordinates $\mathbf{x}^{n+1}$, providing the new deformation gradient tensor $\mathbf{F}_{n+1}$;

(c) given $w^{n+1}, c^{n+1}, \mathbf{F}_{n+1}$, and $J_{n+1}=\operatorname{det}\left(\mathbf{F}_{n+1}\right)$, solve the variational formulation of the Bidomain system (5) with a first order IMEX method and compute the new electric potentials $v^{n+1}, u_{e}^{n+1}$ with an operator splitting method, consisting of decoupling the parabolic from the elliptic equation.

We remark that, in order to avoid numerical instabilities, in point (b), the finite elasticity equations and the active tension model are solved in a coupled way using the update method proposed in Refs. 51 and 52, i.e., the variables of the active tension model are updated at each Newton iteration together with the solution of the mechanical deformation.

In our simulations, the electrical time step size is $\Delta_{e} t$ $=0.05 \mathrm{~ms}$, while the mechanical times step is $\Delta_{m} t=0.25 \mathrm{~ms}$. We remark that to approximate the convective term in the variational formulation of (5), an upwind computation of the nodal gradient of $v^{n}$ is derived, projected onto the deformation 
rate $\frac{\mathbf{x}^{n+1}-\mathbf{x}^{n}}{\Delta t_{n}}$ vector and then integrated against the test function. We refer to Ref. 10 for more details about the numerical scheme.

\section{Computational kernels and parallel solvers}

Due to the employed space and time discretization strategies, at each time step, the main computational kernels are (i) solving the non-linear system deriving from the discretization of the mechanical problem (1) by a parallel Newton-GMRES-Algebraic Multigrid method, see Ref. 9; an alternative Newton-GMRES-BDDC solver could be used as well, see Ref. 54;

(ii) solving the two linear systems deriving from the discretization of the elliptic and parabolic equations in the Bidomain model (5) by a parallel Conjugate Gradient
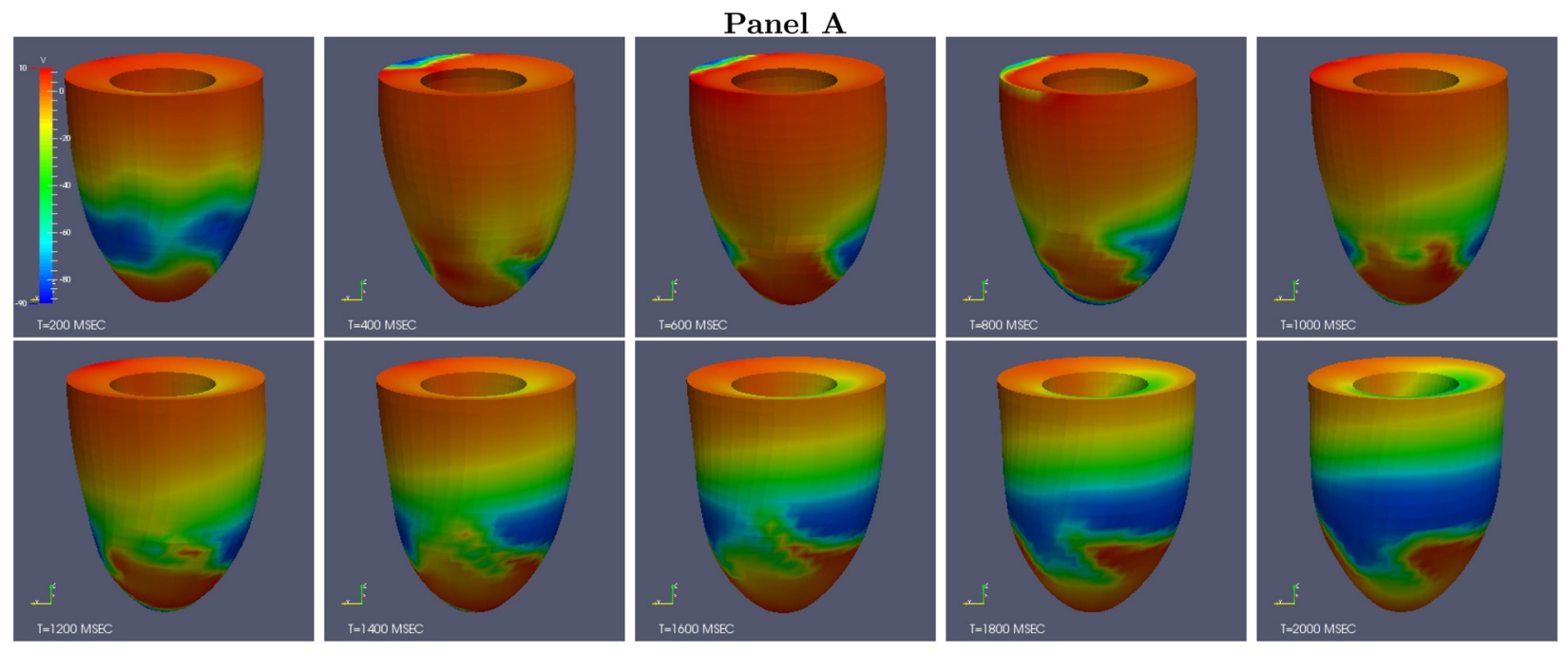

Panel B

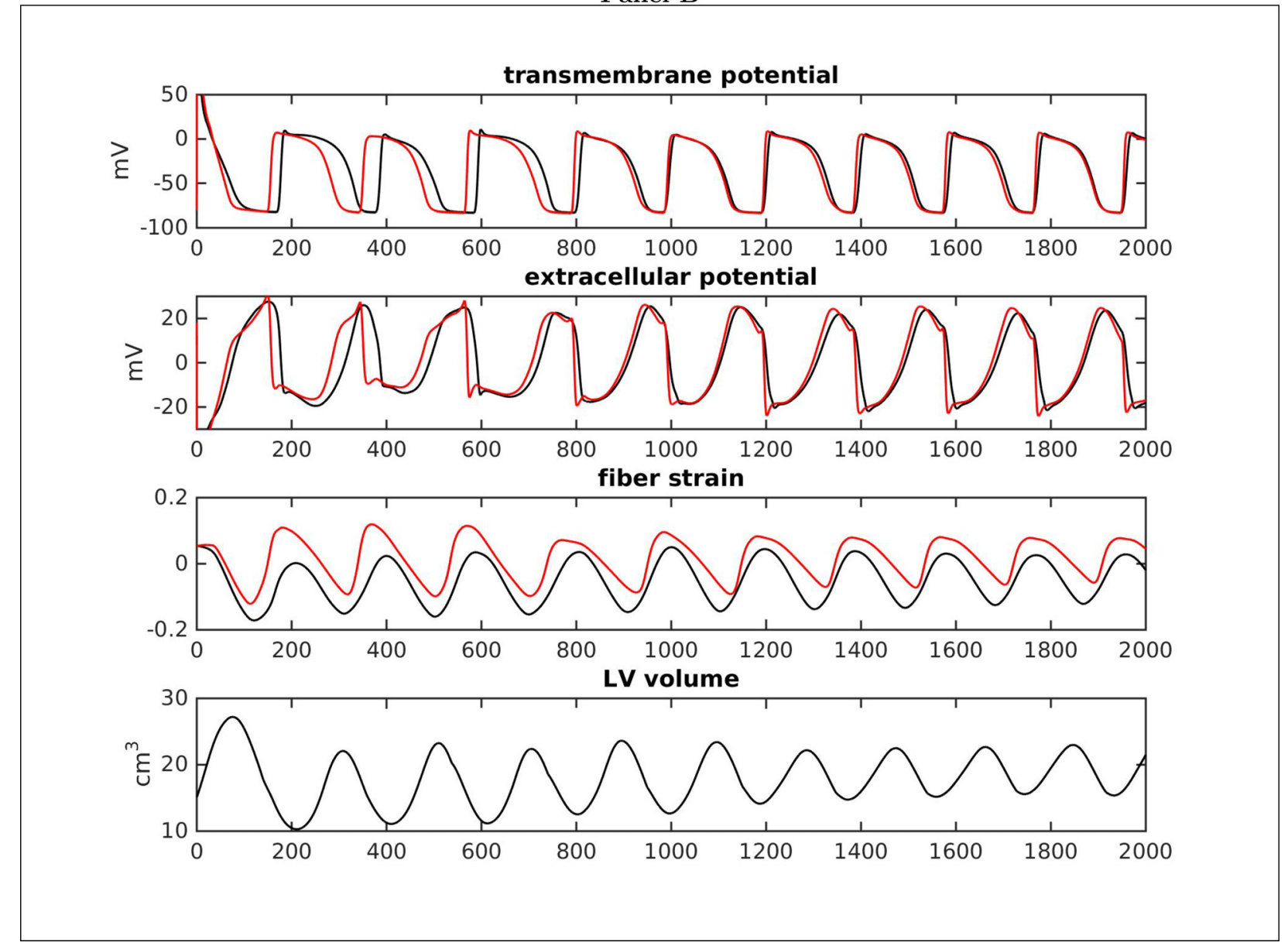

FIG. 2. Simulation SE1: slope 1.8, GEF, noMEF. Panel A: snapshots of the transmembrane potential $v$ every $200 \mathrm{~ms}$ from 200 to $2000 \mathrm{~ms}$. Panel B: waveforms of transmembrane potential, extracellular potential, fiber strain, LV volume computed from an epicardial (black), and an endocardial (red) site. 
method preconditioned with a Multilevel Additive Schwarz preconditioner studied in Ref. 53.

Our parallel simulations have been performed on a Linux cluster using the parallel library $\mathrm{PETSc}^{3}$ from the Argonne National Laboratory, while visualization of the results has been obtained with Paraview and Matlab software.

\section{Parameters calibration}

a. Mechanical model. The parameters of the transversely isotropic strain energy function are adapted from Ref. 14. The bulk modulus is $K=200 \mathrm{kPa}$.

b. Active tension model. The active tension model and its parameters are as in Land et al. ${ }^{41}$

c. Bidomain model. The values of the transversely isotropic conductivity coefficients in (7), that we use in all the numerical tests, are $\sigma_{l}^{i}=3, \sigma_{t}^{i}=0.31525, \sigma_{l}^{e}=2, \sigma_{t}^{e}=1.3514$, all expressed in $\mathrm{m} \Omega^{-1} \mathrm{~cm}^{-1}$. These values, coupled with the TP06 membrane model, predict conduction velocities of about 0.061 and $0.027 \mathrm{~cm} / \mathrm{ms}$ for excitation wavefronts propagating along and across the fiber direction, respectively. We remark that these conduction velocities are within the physiological range. The membrane surface to volume ratio is $\chi=10^{3} \mathrm{~cm}^{-1}$ and the membrane capacitance per unit volume is $c_{m}=\chi C_{m}$, where $C_{m}=1 \mu \mathrm{F} / \mathrm{cm}^{2}$ is the membrane capacitance per unit area.

d. TP06 membrane model. We consider two calibrations of the TP06 model proposed in Ref. 64 (Table 2), one denoted by "slope 1.1" with the four conductivity parameters $G_{K r}=0.153, G_{K s}=0.392, G_{p C a}=0.1238, G_{p K}=0.0146$ set to their normal values and yielding an APD-DI restitution curve with maximum slope 1.1, and the other denoted by "slope 1.8" with conductivity parameters $G_{K r}=0.172, G_{K s}$ $=0.441, G_{p C a}=0.8666, G_{p K}=0.00219$ and doubled $\tau_{f}$ parameter, corresponding to a more arrhythmogenic calibration with a steeper APD-DI restitution curve with a maximum slope 1.8 .

\section{Initial and boundary conditions}

The initial conditions for the electrical model are at resting values for all the potentials and gating variables of the ten Tusscher model, while the boundary conditions for the Bidomain model are for insulated tissue. For what concerns the mechanical boundary conditions, in order to prevent rigid body motion, we considered both setting to zero the displacement at the basal surface similarly to previous studies, ${ }^{23,36}$ and the weaker condition setting to zero the displacement only at the circumferential basal line meeting the endocardial surface, while setting to zero only the third displacement component on the rest of the basal surface. In this work, we report only the results obtained with this weaker basal boundary condition. Moreover, on the endocardial surface, we impose as in Ref. 35 Neumann boundary conditions given by a constant intracavitary blood pressure amounting to $2 \mathrm{kPa}$, and we assume that the rest of domain is not subject to any external loading, i.e., homogeneous Neumann boundary conditions are prescribed elsewhere.
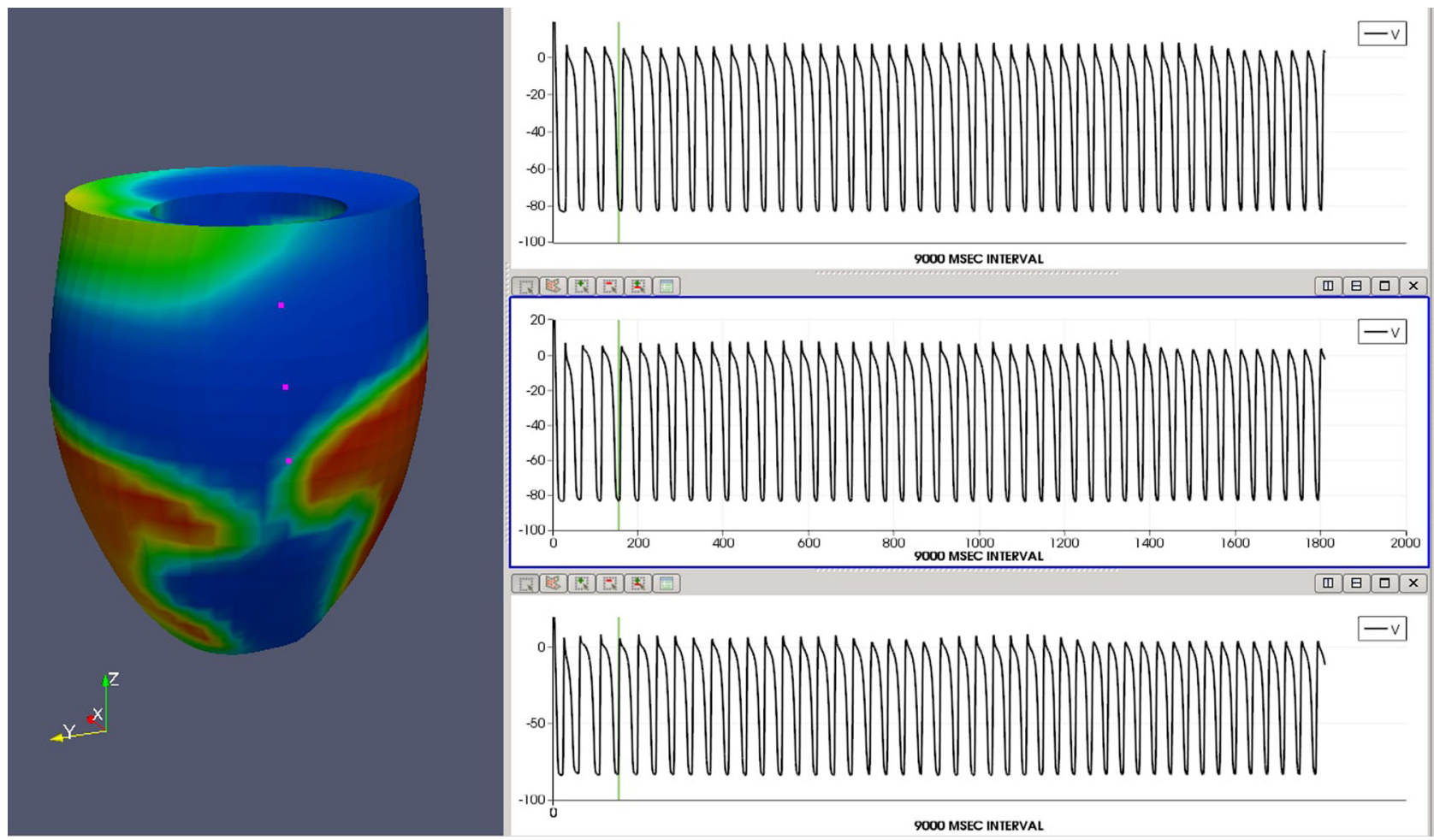

FIG. 3. Simulation SE1: slope 1.8, GEF, noMEF. Longer version up to $9000 \mathrm{~ms}$. Left panel: snapshot of the transmembrane potential after 3 scroll wave rotations (about $155 \mathrm{~ms}$ ). Right panels: Plot of the transmembrane potential over the $9000 \mathrm{~ms}$ simulation interval at the 3 locations marked by red dots in the left panel. 


\section{Geometric (GEF) and mechano-electric (MEF) feedback}

We remark that the influence of the cardiac tissue deformation on the Bidomain model (5) is due to three different mechano-electric influences:

(i) the presence of the deformation gradient $\mathbf{F}$ in the conductivity coefficients structure, i.e., $J \mathbf{F}^{-1} \hat{D}_{i, e} \mathbf{F}^{-T}$, introducing a strain-induced tissue conductivity; (ii) the presence of the deformation gradient $\mathbf{F}$ and the deformation rate $\mathbf{V}$ in the convective term $\mathbf{F}^{-T} \mathrm{Grad} \hat{v} \cdot \mathbf{V}$;

(iii) the presence of the stretch $\lambda$ in the ionic membrane current $i_{\text {ion }}(\hat{v}, \hat{\mathbf{w}}, \hat{\mathbf{c}}, \lambda)$ due to the stretch-activated current $I_{s a c}$, also known as mechano-electric feedback.

In the following, we will refer to (i) and (ii) as geometric feedback (GEF) and to (iii) as mechano-electric feedback (MEF).
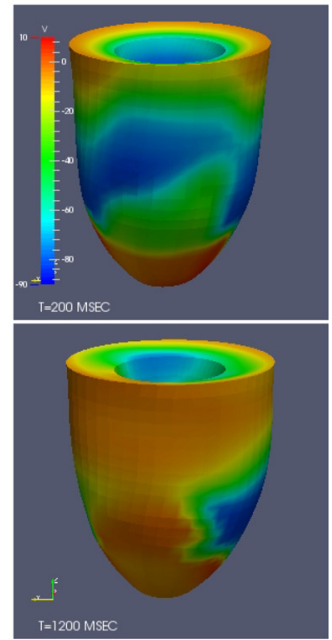
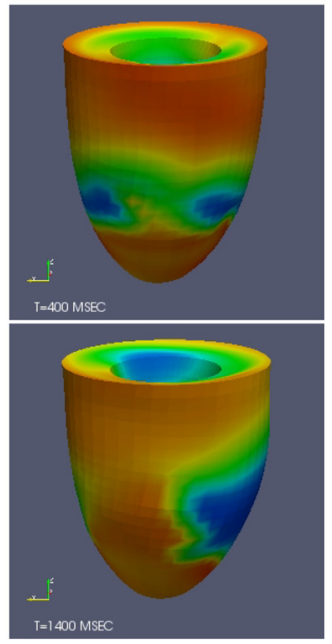

\section{Panel A}
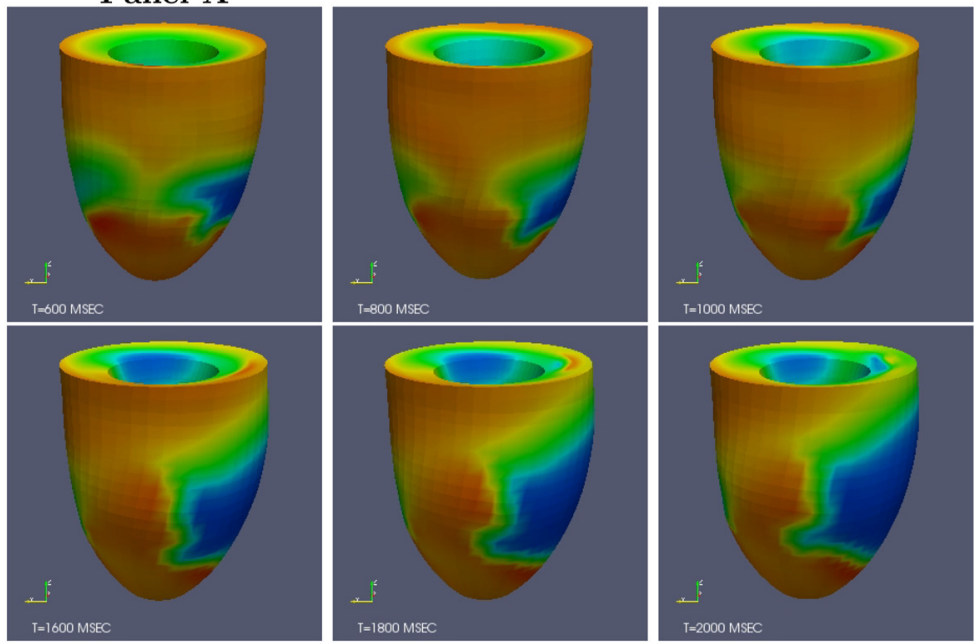

Panel B
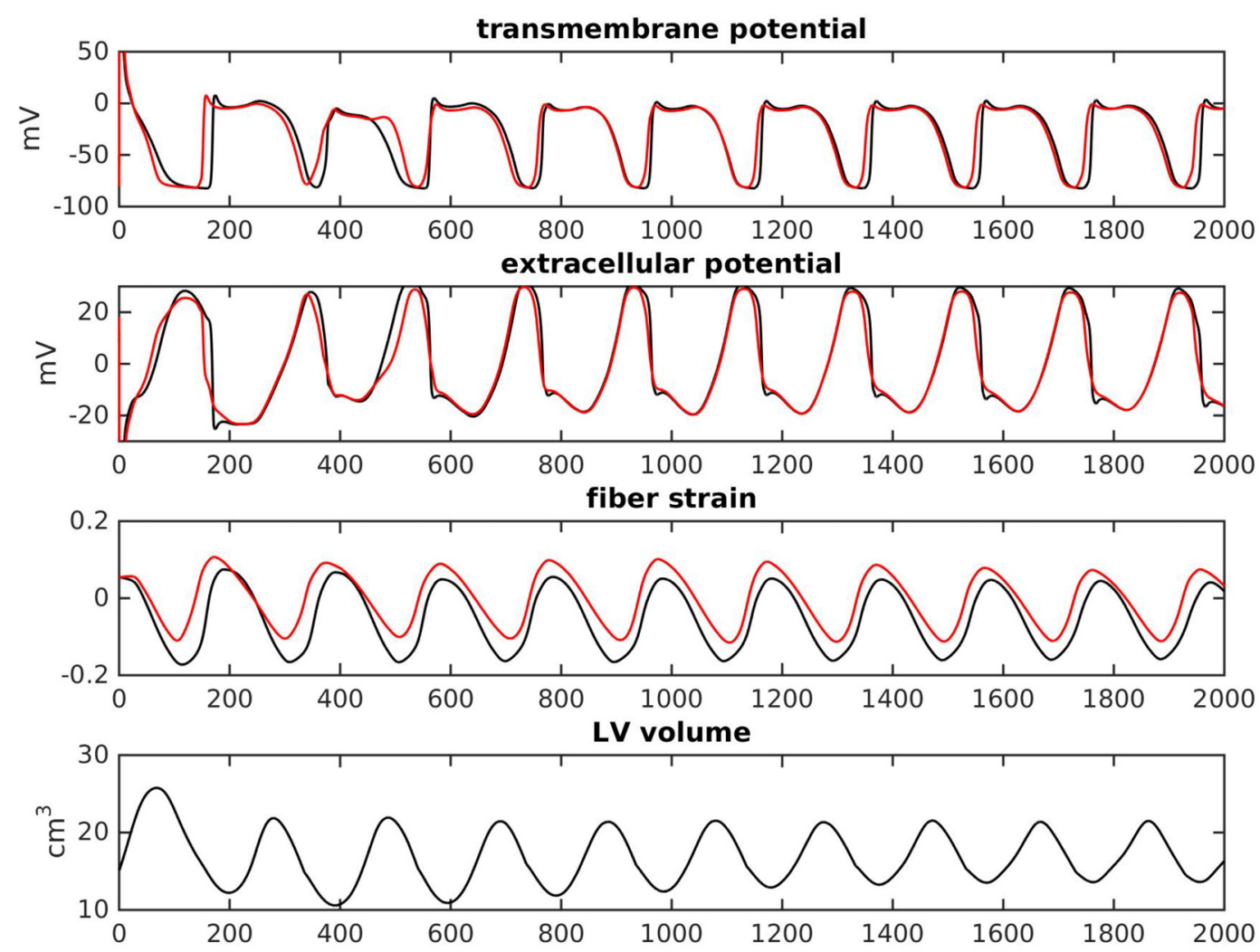

FIG. 4. Simulation SE2: slope 1.8, GEF, MEF, $v_{n s}=-10 \mathrm{mV}$. Same format as in Fig. 2. 


\section{S1-S2 cross-gradient initiation of re-entry and electro-mechanical simulations}

We first paced our cardiac electromechanical model with four apical stimulations at a basic cycle length (CBL) of $350 \mathrm{~ms}$, yielding excitation wavefronts propagating from the apex to the base of the idealized left ventricle.

At $280 \mathrm{~ms}$ after the fourth $\mathrm{S} 1$ stimulus is delivered, we apply a premature $\mathrm{S} 2$ cross-gradient stimulation current from the base to the apex and across the wall thickness, covering about a third of ventricular volume, to induce a ventricular reentry consisting of a pair of counter-rotating scroll waves. We run the simulation for $2000 \mathrm{~ms}$ after the $\mathrm{S} 2$ delivery, in the following five settings:

- SE1: slope 1.8, GEF, noMEF. Membrane parameters set according to calibration slope 1.8 , both geometric feedback (i) and (ii) are included, and stretch-activated currents are neglected;
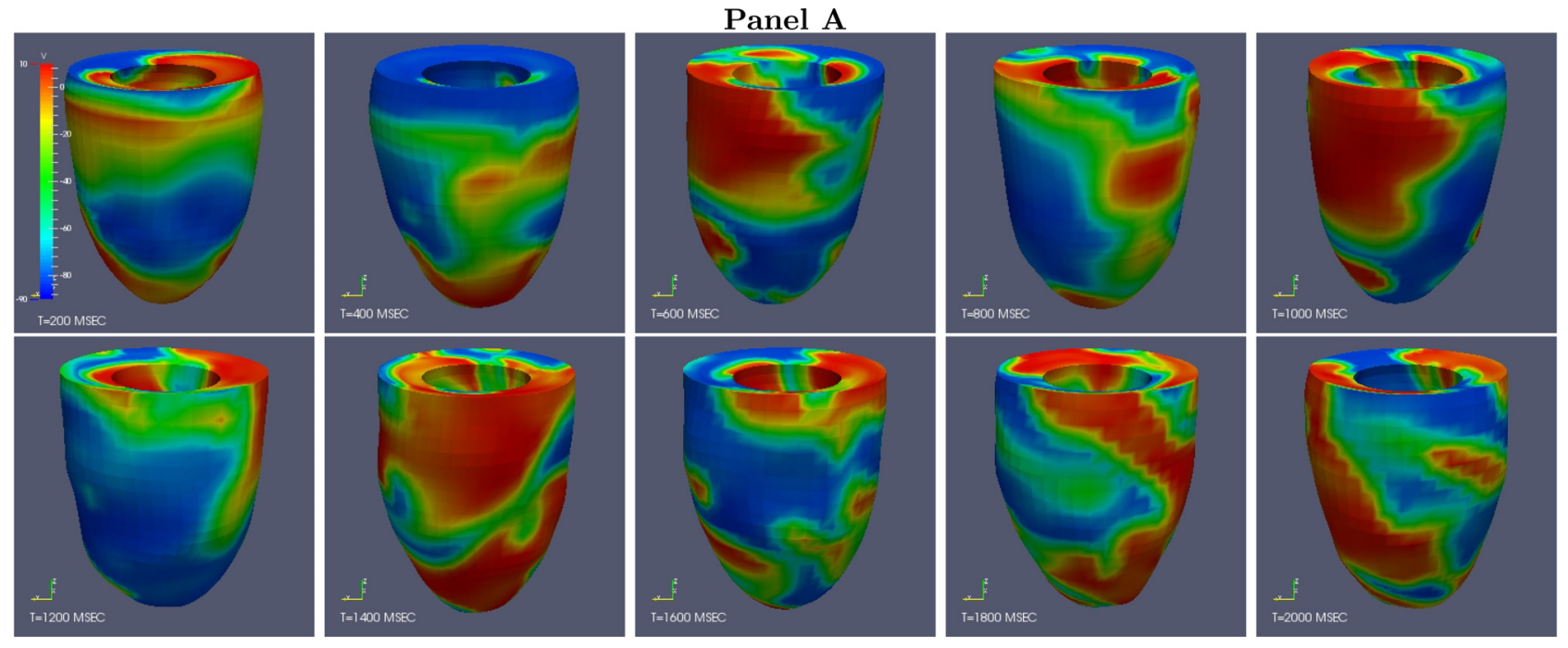

Panel B
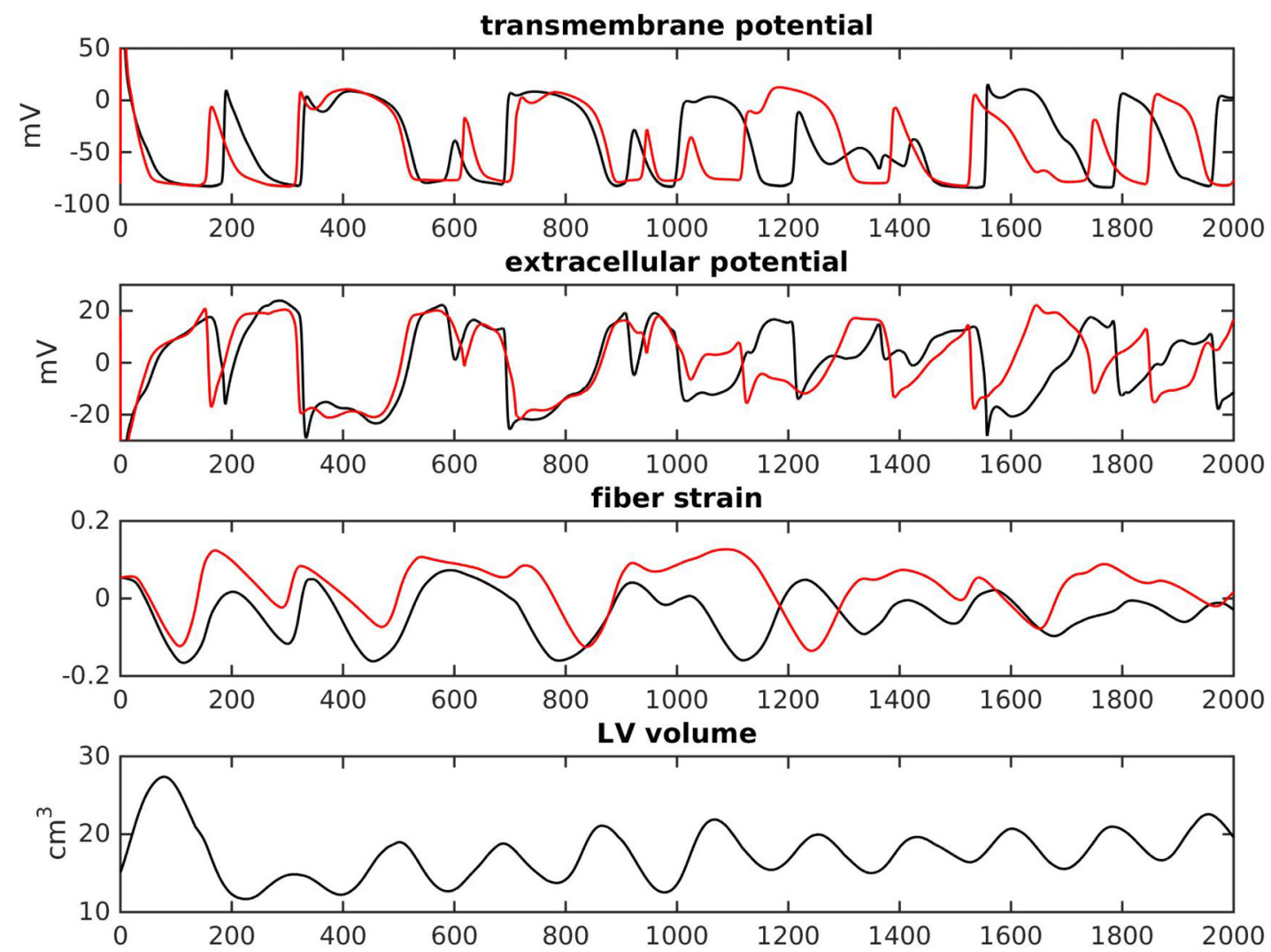

FIG. 5. Simulation SE3: slope 1.8, GEF, MEF, $v_{n s}=-60 \mathrm{mV}$. Same format as in Fig. 2. 
- SE2: slope 1.8, GEF, MEF, $v_{n s}=-\mathbf{1 0} \mathrm{mV}$. Membrane parameters set according to calibration slope 1.8 , both geometric feedback (i) and (ii) are included, and stretchactivated currents are included with $v_{n s}=-10 \mathrm{mV}$;

- SE3: slope 1.8, GEF, MEF, $v_{n s}=-60 \mathrm{mV}$. Membrane parameters set according to calibration slope 1.8, both geometric feedback (i) and (ii) are included, and stretchactivated currents are included with $v_{n s}=-60 \mathrm{mV}$;

- SE4: slope 1.1, GEF, MEF, $v_{n s}=-10 \mathrm{mV}$. Membrane parameters set according to calibration slope 1.1, both geometric feedback (i) and (ii) are included, and stretchactivated currents are included with $v_{n s}=-10 \mathrm{mV}$;

- SE5: slope 1.1, GEF, MEF, $v_{n s}=-60 \mathrm{mV}$. Membrane parameters set according to calibration slope 1.1, both geometric feedback (i) and (ii) are included, and stretchactivated currents are included with $v_{n s}=-60 \mathrm{mV}$.

\section{RESULTS}

In order to elucidate the influence of GEF and MEF on the stability of starting scroll waves with two filaments, in Figs. 2, 4, 5, 7, and 8, we report, for each of the previous five simulation settings SE1-5, the snapshots taken every $200 \mathrm{~ms}$ of the transmembrane potential distribution on the deforming ventricle and the waveforms at selected epicardial points of the transmembrane potential $v$, extracellular potential $u_{e}$, fiber strain $\left(\lambda^{2}-1\right) / 2$, and intracavitary volume.

\section{A. SE1: Slope 1.8, GEF, noMEF}

We first consider the arrhythmogenic substrate related to the TP06 model with maximum APD restitution slope 1.8, neglecting the presence of $I_{s a c}$. The two scroll waves generated by the S2 stimulus continue to rotate without breaking, leading to a stable ventricular tachycardia pattern. Indeed, all the waveforms displayed in Fig. 2 exhibit a very regular periodic behavior, with a fast beating rate. During the $2000 \mathrm{~ms}$ interval considered, the transmembrane potential waveforms display 10 complete action potentials and the beginning of an 11th one. We have run this simulation also for an extended time interval of $9000 \mathrm{~ms}$ and observed the same regular dynamics (see Fig. 3). We have computed the APD of the first two beats elicited after the initiation of reentry, from 27 epicardial sites located in the half of the idealized ventricle where the S2 stimulation is delivered. The average APD of the first beat amounts to $110 \mathrm{~ms}(\min =38 \mathrm{~ms}, \max =153 \mathrm{~ms})$, with a dispersion $(=\max -\min )$ of $115 \mathrm{~ms}$. The values computed from the second beat are analogous.

\section{B. SE2: Slope 1.8, GEF, MEF, $v_{n s}=-10 \mathrm{mV}$}

In this setting, we keep all the parameters as in the previous SE1 setting, but we introduce the $I_{s a c}$ current with $v_{n s}=-10 \mathrm{mV}$, yielding a high $I_{s a c}$ reversal potential of about $-20 \mathrm{mV}$. The transmembrane potential snapshots on the deforming ventricle reported in Fig. 4 show that the two scroll waves generated by the S2 stimulus, as in the SE1 simulation, rotate regularly without breaking, leading to ventricular tachycardia. All the waveforms displayed in Fig. 4 exhibit the same periodic behavior of the SE1 case. As in the previous simulation, we have computed the APD of the first two beats elicited after the initiation of reentry. The average APD of the first beat is $123 \mathrm{~ms}(\mathrm{~min}=61 \mathrm{~ms}, \max =172 \mathrm{~ms})$,
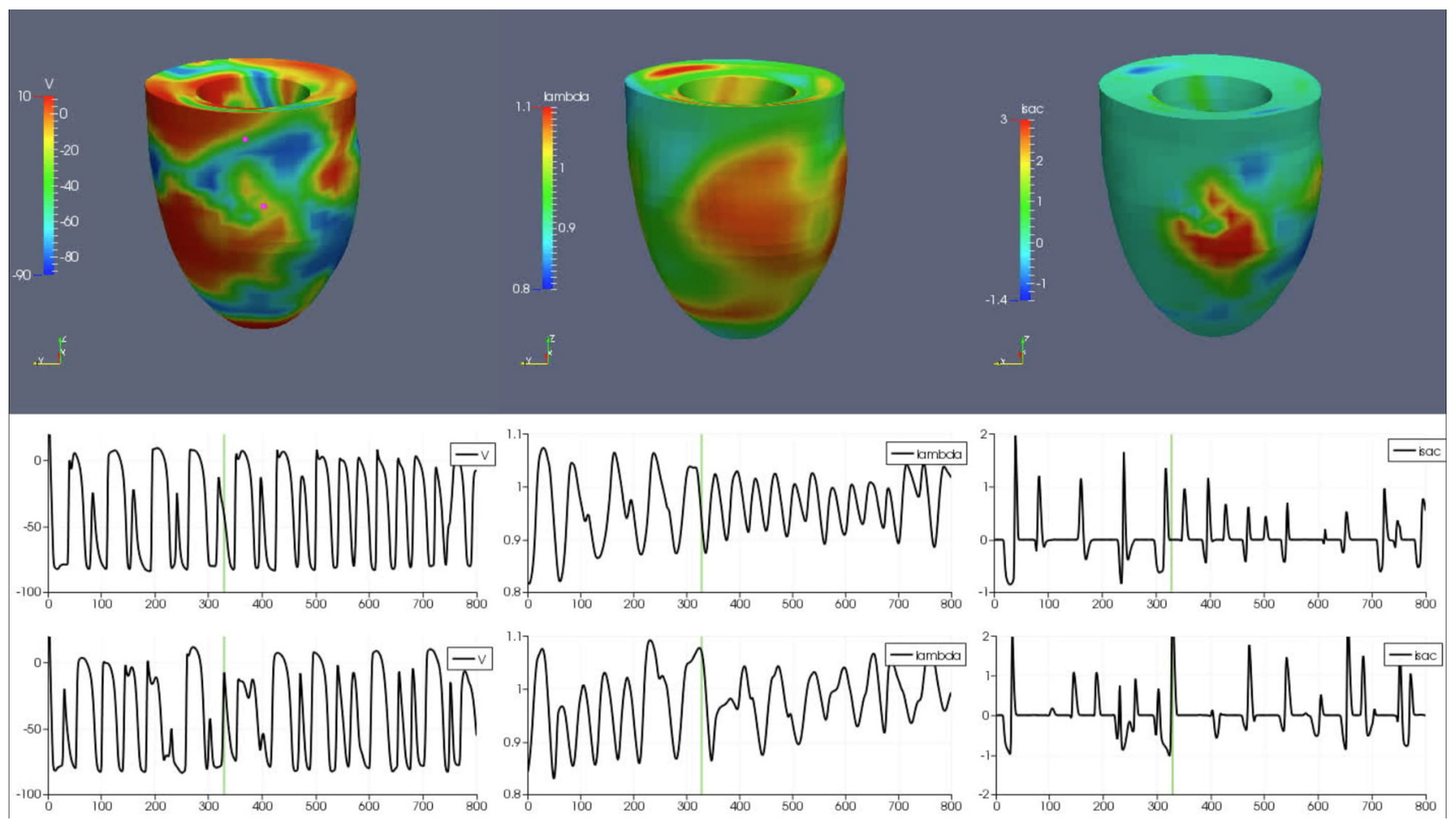

FIG. 6. Simulation SE3 over $4000 \mathrm{~ms}$ : slope 1.8, GEF, MEF, $v_{n s}=-60 \mathrm{mV}$. Top: distribution of the transmembrane potential $v$, stretch $\lambda$, and stretch-activated current $I_{s a c}$ at the time instant indicated by the green bar in the waveforms below. Bottom: waveforms of $v, \lambda$, and $I_{s a c}$ at the two epicardial points indicated by the two red dots in the top left $v$ distribution plot. 
with a dispersion of $111 \mathrm{~ms}$. Again the values computed from the second beat are analogous.

\section{SE3: Slope 1.8, GEF, MEF, $v_{n s}=-60 \mathrm{mV}$}

In this test, we change the $I_{s a c}$ current by setting the parameter $v_{n s}=-60 \mathrm{mV}$, yielding a low $I_{s a c}$ reversal potential of about $-60 \mathrm{mV}$. After the first rotation, the two scroll waves break up into several smaller scroll waves and generate irregular transmembrane potential distributions characterized by high electrical turbulence, often associated with ventricular fibrillation, as shown in the snapshots of Fig. 5. This turbulence is confirmed by the irregular time evolution of the transmembrane and extracellular potential waveforms, as well as the fiber strain. The LV volume still show some oscillations but of reduced amplitude. Thus, the combined role of arrhythmogenic substrate and low $I_{s a c}$ reversal potential seems to induce deterioration of the stability of scroll waves,
Panel A
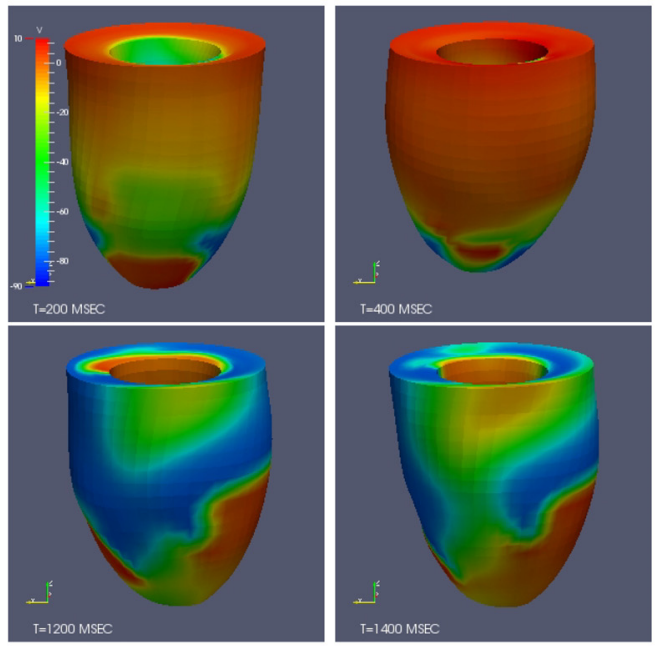
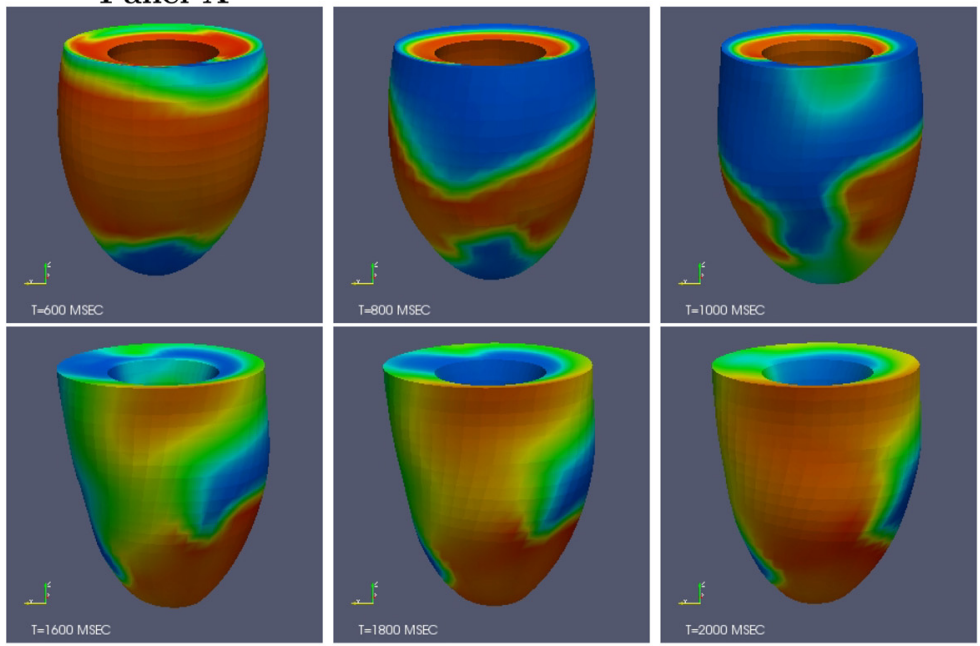

Panel B
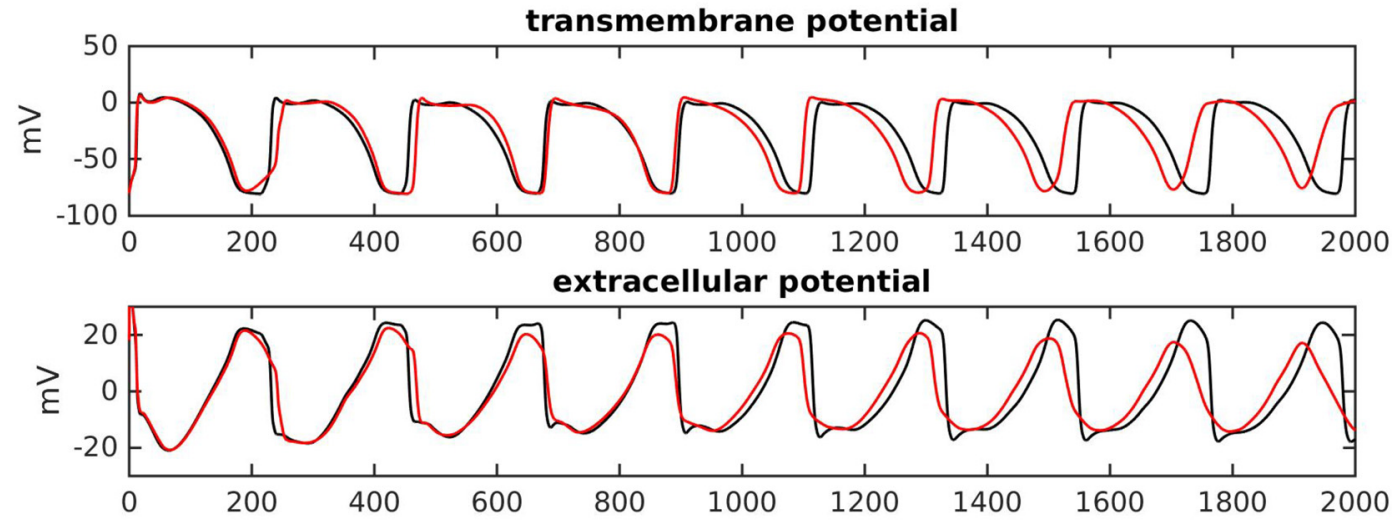

fiber strain

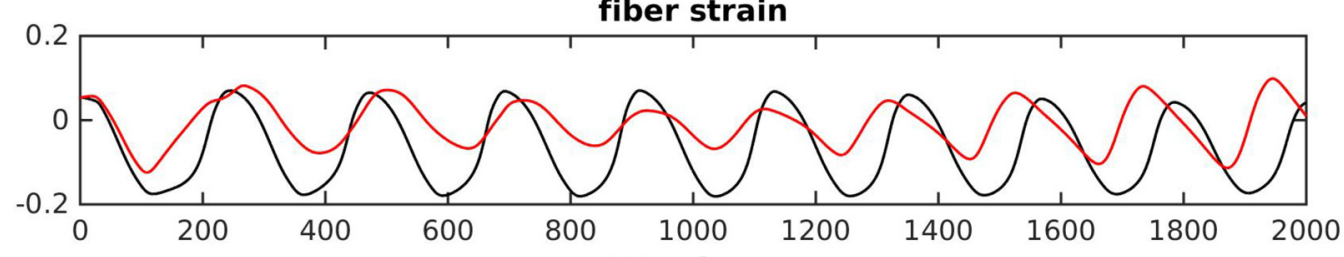

LV volume

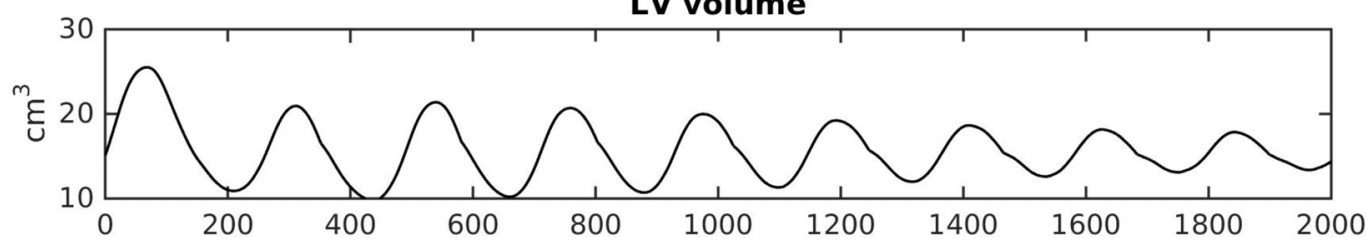


promoting the onset of ventricular fibrillation. Differently from simulations SE1 and SE2, the computation of APD from the first two beats in the 27 epicardial sites shows the presence of APD alternans. In fact, the average APD of the first beat is $56 \mathrm{~ms}(\mathrm{~min}=10 \mathrm{~ms}, \max =160 \mathrm{~ms})$, with a dispersion of $150 \mathrm{~ms}$, while the average APD of the second beat is $143 \mathrm{~ms}(\min =18 \mathrm{~ms}, \max =218 \mathrm{~ms})$, with a dispersion of $200 \mathrm{~ms}$.
This is further studied in Fig. 6 over a longer time interval of $4000 \mathrm{~ms}$ ( 800 time steps of $5 \mathrm{~ms}$ each). In the top row of this figure, we report the distribution of the transmembrane potential $v$, stretch $\lambda$, stretch-activated current $I_{s a c}$ at the time instant indicated by the green bar in the waveforms below, while in the bottom row we report the waveforms of $v, \lambda, I_{s a c}$ at the two epicardial points indicated by the two pink dots in the $v$ distribution plot above. The reported
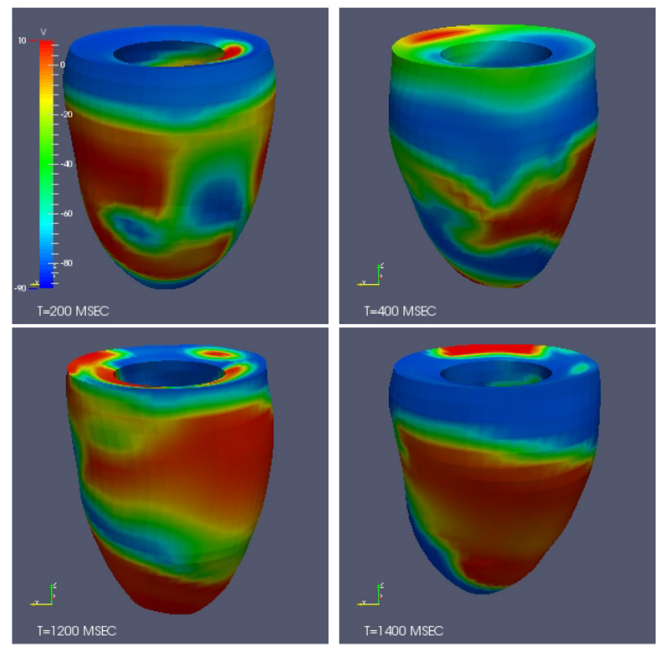

\section{Panel A}
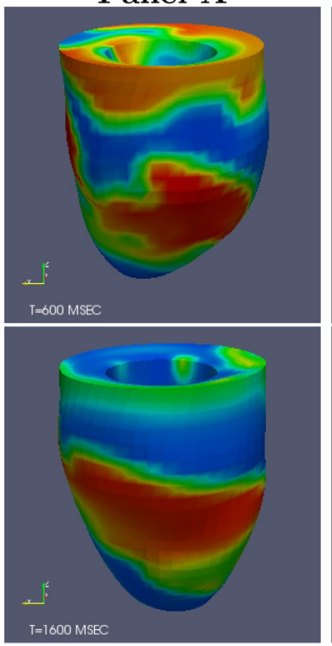
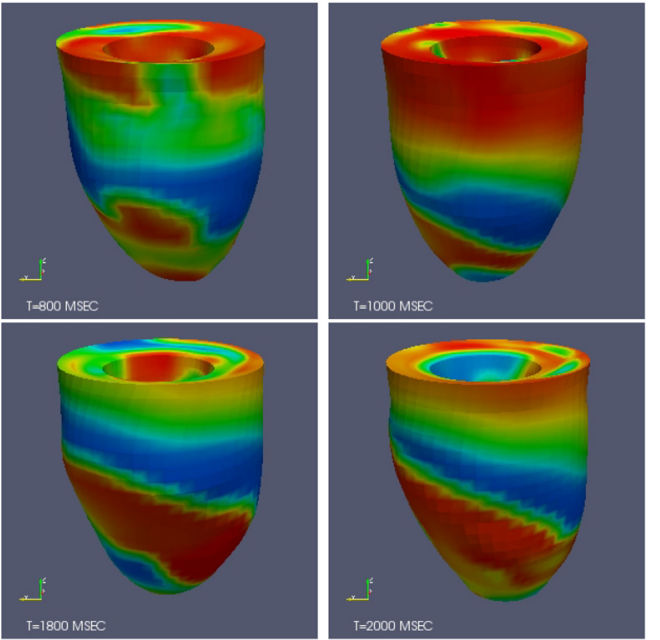

Panel B
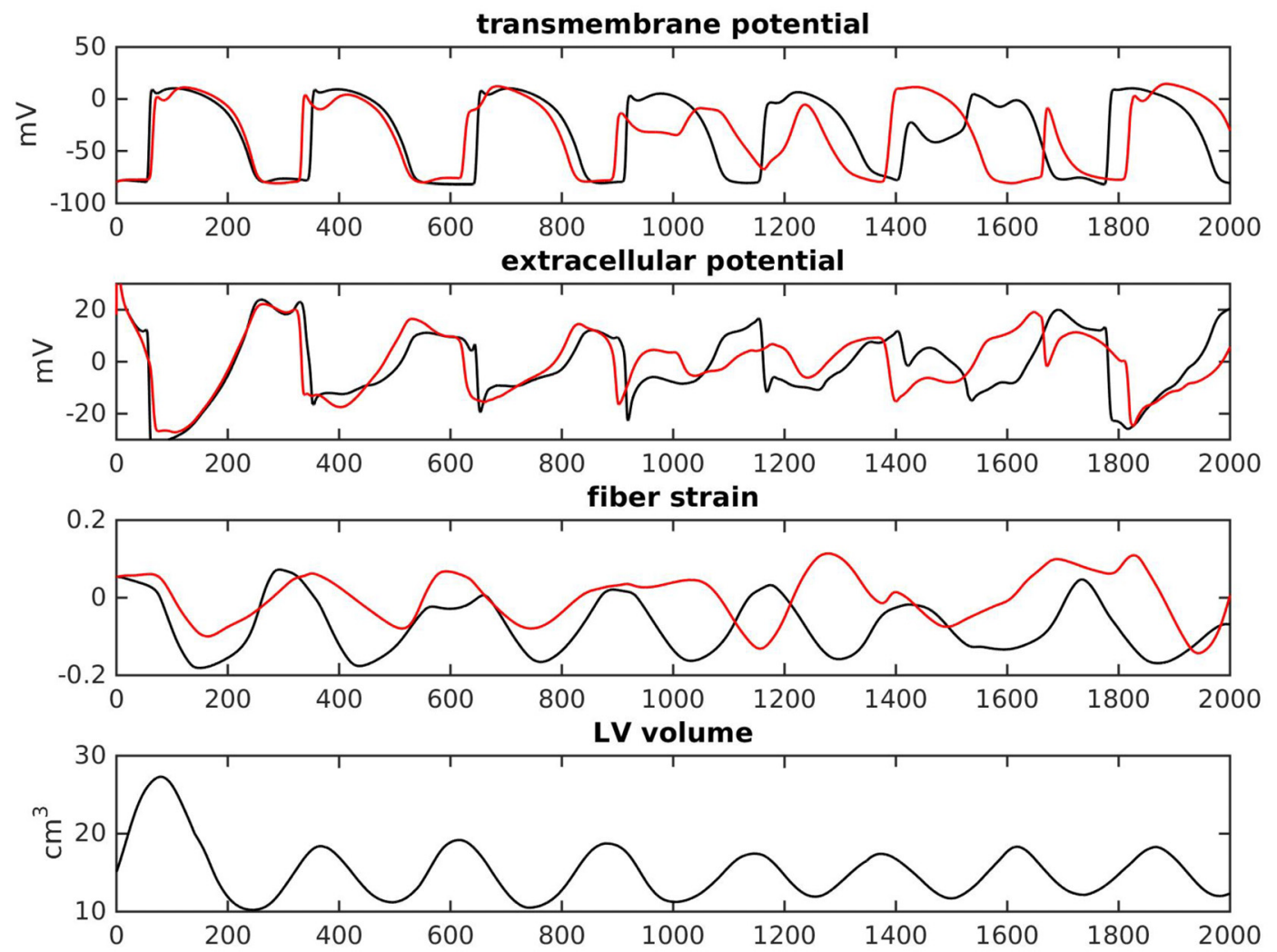
waveforms show that when the stretch $\lambda$ is greater than one (e.g., at the time indicated by the green bar) there is a positive spike of the $I_{s a c}$ current yielding a repolarizing current which induces electrical alternances of short (triangular) and regular action potentials.

\section{SE4: Slope 1.1, GEF, MEF, $v_{n s}=-10 \mathrm{mV}$}

We then reconsider the original stretch-activated current $I_{s a c}$ with $v_{n s}=-10 \mathrm{mV}$ but in the slope 1.1 parameter calibration of the TP06 model. This calibration has been shown to be less arrhythmogenic than the slope 1.8 calibration, because the restitution curve is less steep; see Ref. 64. As in simulation SE2, a $I_{s a c}$ reversal potential of about $-20 \mathrm{mV}$ yields a stable ventricular tachycardiac pattern, with two scroll waves rotating with a regular rhythm, as shown in Fig. 7. The period is slightly slower than in the SE2 setting, since the waveforms display 9 instead of 10 complete action potentials during the $2000 \mathrm{~ms}$ interval.

\section{E. SE5: Slope 1.1, GEF, MEF, $v_{n s}=-60 \mathrm{mV}$}

In this final test, we keep all the parameters as in the SE4, except that we change the $I_{s a c}$ current by setting the parameter $v_{n s}=-60 \mathrm{mV}$, yielding a low $I_{s a c}$ reversal potential of about $-60 \mathrm{mV}$. The snapshots of Fig. 8 show that the two scroll waves generated by the S2 stimulus, after an initial stable periodic regime, around $800 \mathrm{~ms}$ break up as in the SE3 setting, leading to a ventricular fibrillation pattern. The dynamics appears less turbulent than in the SE3 setting with slope 1.8 , but it is still quite irregular after $1000 \mathrm{~ms}$, as shown by the waveforms displayed in Fig. 8 .

\section{DISCUSSION}

We have investigated, by means of three-dimensional simulations based on a strongly coupled cardiac electromechanical coupling model, the influence of ventricular deformation on the maintenance of ventricular tachycardia and fibrillation. Previous simulations studies focusing on the effects of mechanical feedback on the generation and stability of cardiac arrhythmias have considered reduced electrical current flows model, such as the Monodomain equation, and simplified stretch-activated current models.

The main novelty of the present work is the extension of these previous pioneering studies in order to include in the electro-mechanical coupling model the following components: the Bidomain representation of the electrical current flow; a convective term in the Bidomain equations, that takes into account the effect of deformation rate; the stretch-activated current model developed by Niederer and Smith in Ref. 50, which consists of both non-selective and potassium selective components and it was validated by fitting experimental data.

The results of our 3D Bidomain electro-mechanical simulations indicate that the stability of cardiac scroll waves induced by $\mathrm{S} 1-\mathrm{S} 2$ cross gradient stimulations is influenced by the mechano-electric feedback induced by the stretchactivated current $I_{s a c}$. In particular, lowering the $I_{s a c}$ resting potential to $v_{n s}=-60 \mathrm{mV}$ yields scroll wave breakups that generate turbulent dynamics in the electrical and mechanical variables, in both the slope 1.1 (normal) and slope 1.8 (arrhythmogenic) calibrations of the TP06 membrane model. In the latter case, the computation of APD from several epicardial sites have shown that scroll wave breakup might be due to the presence of APD alternans and large APD spatial dispersion. We did not observe scroll wave breakup in either calibrations when $I_{s a c}$ was not included or when the $I_{s a c}$ resting potential was increased to $v_{n s}=-10 \mathrm{mV}$. Without including the mechano-electric feedback, the only presence of geometric feedback increased the scroll wave meandering, but did not determine the scroll wave stability in our simulations.

The effects of mechano-electric feedback on the stability of cardiac scroll waves have been investigated in the previous simulations studies. ${ }^{29,35}$ Although our work and the simulation studies of Refs. 29 and 35 use the same membrane model TP06, we remark that there are some modeling differences in the components of the electromechanical model used in this work compared with those of Refs. 29 and 35: for the electrical current flow model, this work uses the Bidomain model while Refs. 29 and 35 used the Monodomain model; for the stretch activated current, we use the model of Ref. 50, while Refs. 29 and 35 used a linear model; for the active tension generation, in this work, we use the model of Ref. 41, while Ref. 35 used the model of Ref. 49, and Ref. 29 used the model of Ref. 58; for the strain energy function, in this work, we use the transversely isotropic strain energy of Refs. 14 and 28, while Ref. 35 used the transversely isotropic strain energy of Refs. 21 and 29 the orthotropic strain energy of Refs. 22 and 67.

We start comparing our results with those obtained in Ref. 29, based on the TP06 membrane model with APD restitution with a maximal slope 1.8. First, the influence of the stretch-activated current was studied in Ref. 29 by starting with a fibrillation state and investigating when the presence of the $I_{s a c}$ current inhibits the already present scroll wave breakup. On the contrary, in our study, we start with a double stable scroll waves and we investigate when the presence of $I_{s a c}$ generates multiple breakups. Furthermore, a non-selective SAC model, linear in $\lambda$ and $v$, was adopted, with conductance $g_{s}$ varying up to $0.07 \mathrm{mS} / \mathrm{cm}^{2}$. For large values of $g_{s}$ and SAC reversal potential of $-60 \mathrm{mV}$, scroll waves breakup was observed, in agreement with our simulation SE3, where the two original scroll waves degenerate rapidly into a turbulent excitation dynamics. For large values of $g_{s}$ and SAC reversal potential of $-10 \mathrm{mV}$, the results in Ref. 29 showed that multiple scroll waves breakup persists, whereas in our comparable simulation setting SE2, the two scroll waves do not break up, leading to a stable ventricular tachycardia.

We now compare our results with those obtained in Ref. 35 , based on an electro-mechanical monodomain model coupled to the TP06 membrane model with APD restitution with a maximal slope 1.1. A non-selective SAC linear in $\lambda$ and $v$ was adopted, with reversal potential of $-20 \mathrm{mV}$ and conductance varying in a large range. Therefore, we can only compare these results with the results of our simulation SE4, where a similar reversal potential of $\approx-19 \mathrm{mV}$ is displayed by the linear approximation $I_{s}(v)=g_{s} \gamma_{s}(\lambda)\left(v-v_{s a c}\right)$ of our SAC model, but with a value of SAC conductance of about 
$g_{s} \approx 0.01 \mathrm{mS} / \mathrm{cm}^{2}$, quite lower than the values considered in Ref. 35. At low values of SAC conductance $g_{s}$, almost no creation or destruction of filaments was observed in Ref. 35, in agreement with the results of our simulation SE4, where the two scroll waves remain stable during the $2000 \mathrm{~ms}$ simulation interval.

\section{A. Limitations and future works}

In order to avoid masking the effect of the heterogeneities provided by the distributions of the fiber stretch in the SAC current, we have disregarded the heterogeneity of the bioelectric activity of the myocyte across the ventricular wall and in the apex-to-base direction.

Future work should be devoted to investigate the role of orthotropic tissue anisotropy and of the application of a variable endocardial pressure, derived from a lumped model of the pressure-volume loop, instead of transversely isotropic tissue anisotropy and of a constant endocardial pressure.

It could be also interesting to investigate the influence of different boundary conditions for the mechanical model, as well as the presence of a Purkinje network as in Ref. 69, which could affect the S1-S2 cross gradient protocol and subsequent reentry dynamics.

Finally, as observed in the Introduction, the recent use of optical transmembrane potential recordings combined with a tissue tracking techniques, see e.g., Refs. 43, 62, and 73 , would allow the comparison between the optical recording map and the optical transmembrane potentials simulated by using electro-mechanical models. This could help to elucidate the mechanisms underlying mechano-electric feedback and related arrhythmias.

\section{ACKNOWLEDGMENTS}

This work was partially supported by grants of M.I.U.R (PRIN 201289A4LX_002), of Istituto di Matematica Applicata e Tecnologie Informatiche C.N.R., Pavia, Italy, and of Istituto Nazionale di Alta Matematica (INdAM), Italy.

${ }^{1}$ I. Adeniran, J. C. Hancox, and H. Zhang, "Effect of cardiac ventricular mechanical contraction on the characteristics of the ECG: A simulation study," J. Biomed. Sci. Eng. 6, 47-60 (2013).

${ }^{2}$ D. Ambrosi, G. Arioli, F. Nobile, and A. Quarteroni, "Electromechanical coupling in cardiac dynamics: The active strain approach," SIAM J. Appl. Math. 71, 605-621 (2011).

${ }^{3}$ S. Balay, K. Buschelman, W. D. Gropp, D. Kaushik, M. Knepley, L. Curfman McInnes, B. F. Smith, and H. Zhang, PETSc User's Manual, Technical Report ANL-95/11 - Revision 3.3, Argonne National Laboratory, 2012.

${ }^{4}$ M. J. Bishop, B. Rodriquez, J. Eason, J. P. Whiteley, N. Trayanova, and D. J. Gavaghan, "Synthesis of voltage-sensitive optical signals: Application to panoramic optical mapping," Biophys. J. 90, 2938-2945 (2006).

${ }^{5}$ M. J. Bishop, B. Rodriquez, F. Qu, I. R. Efimov, D. J. Gavaghan, and N. A. Trayanova, "The role of photon scattering in optical signal distortion during arrhythmia and defrillation," Biophys. J. 93, 3714-3726 (2007).

${ }^{6} \mathrm{E}$. M. Cherry and F. H. Fenton, "Suppression of alternans and conduction blocks despite steep APD restitution: Electrotonic, memory, and conduction velocity restitution effects," Am. J. Physiol. Heart Circ. Physiol. 286, H2332-H2341 (2004).

${ }^{7}$ P. Colli Franzone, L. Guerri, M. Pennacchio, and B. Taccardi, "Spread of excitation in 3-D models of the anisotropic cardiac tissue. II. Effects of fiber architecture and ventricular geometry," Math. Biosci. 147, 131-171 (1998).
${ }^{8}$ P. Colli Franzone, L. F. Pavarino, and S. Scacchi, Mathematical Cardiac Electrophysiology (Springer, MSA, New York, 2014), Vol. 13.

${ }^{9}$ P. Colli Franzone, L. F. Pavarino, and S. Scacchi, "Parallel multilevel solvers for the cardiac electro-mechanical coupling," Appl. Numer. Math. 95, 140-153 (2015).

${ }^{10}$ P. Colli Franzone, L. F. Pavarino, and S. Scacchi, "Bioelectrical effects of mechanical feedbacks in a strongly coupled cardiac electro-mechanical model," Math. Mod. Methods Appl. Sci. 26(1), 27-57 (2016).

${ }^{11}$ P. Colli Franzone, L. F. Pavarino, and S. Scacchi, "Joint influence of transmural heterogeneities and wall deformation on cardiac bioelectrical activity: A simulation study," Math. Biosci. 280, 71-86 (2016).

${ }^{12}$ J. Constantino, Y. X. Hu, A. C. Lardo, and N. A. Trayanova, "Mechanistic insight into prolonged electromechanical delay in dyssynchronous heart failure: A computational study," Am. J. Physiol. Heart Circ. Physiol. 305, H1265-H1273 (2013).

${ }^{13}$ B. L. de Oliveira, B. M. Rocha, L. P. S. Barra, E. M. Toledo, J. Sundnes, and R. Weber dos Santos, "Effects of deformation on transmural dispersion of repolarization using in silico models of human left ventricular wedge," Int. J. Numer. Methods. Biomed. Eng. 29, 1323-1337 (2013).

${ }^{14}$ T. S. E. Eriksson, A. J. Prassl, G. Plank, and G. A. Holzapfel, "Influence of myocardial fiber/sheet orientations on left ventricular mechanical contraction," Math. Mech. Solids 18, 592-606 (2013).

${ }^{15}$ M. Favino, S. Pozzi, S. Pezzuto, F. W. Prinzen, A. Auricchio, and R. Krause, "Impact of mechanical deformation on pseudo-ECG: A simulation study," Europace 18, 77-84 (2016).

${ }^{16}$ F. J. Fenton, E. M. Cherry, H. M. Hastings, and S. J. Evans, "Multiple mechanisms of spiral wave breakup in a model of cardiac electrical activity," Chaos 12, 852-892 (2002).

${ }^{17}$ F. J. Fenton and A. Karma, "Vortex dynamics in three-dimensional continuous myocardium with fiber rotation: Filament instability and fibrillation," Chaos 8, 20-47 (1998).

${ }^{18}$ T. Fritz, C. Wieners, G. Seemann, H. Steen, and O. Doessel, "Simulation of the contraction of the ventricles in a human heart model including atria and pericardium," Biomech. Mod. Mechanobiol. 13(3), 627-641 (2014).

${ }^{19} \mathrm{~S}$. Göktepe and E. Kuhl, "Electromechanics of the heart - a unified approach to the strongly coupled excitation-contraction problem," Comput. Mech. 45, 227-243 (2010).

${ }^{20}$ R. A. Gray, A. M. Pertson, and J. Jalife, "Spatial and temporal organization during cardiac fibrillation," Nature 392, 75-78 (1998).

${ }^{21}$ J. M. Guccione, A. D. McCulloch, and L. K. Waldman, "Passive material properties of intact ventricular myocardium determined from a cylindrical model," J. Biomech. Eng. 113, 42-55 (1991).

${ }^{22}$ J. M. Guccione, K. D. Costa, and A. D. McCulloch, "Finite element stress analysis of left ventricular mechanics in the beating dog heart," J. Biomech. 28, 1167-1177 (1995).

${ }^{23}$ V. Gurev, J. Constantino, J. J. Rice, and N. A. Trayanova, "Distribution of electromechanical delay in the heart: Insights from a three-dimensional electromechanical model," Biophys. J. 99, 745-754 (2010).

${ }^{24}$ V. Gurev, T. Lee, J. Constantino, H. Arevalo, and N. A. Trayanova, "Models of cardiac electromechanics based on individual hearts imaging data: Image-based electromechanical models of the heart," Biomech. Model. Mechanobiol. 10, 295-306 (2011).

${ }^{25}$ C. J. Hyatt, S. F. Mironov, M. Wellner, O. Berenfeld, A. K. Popp, D. A. Weiz, J. Jalife, and A. M. Pertsov, "Synthesis of voltage-sensitive fluorescence signals from three-dimensional myocardium activation patterns," Biophys. J. 85, 2673-2683 (2003).

${ }^{26}$ S. N. Healy and A. D. McCulloch, "An ionic model of stretch-activated and stretch-modulated currents in rabbit ventricular myocytes," Europace 7, S128-S134 (2005).

${ }^{27}$ T. J. Herron, P. Lee, and J. Jalife, "Optical imaging of voltage and calcium in cardiac cells \& tissues," Circ. Res. 110, 609-623 (2012).

${ }^{28}$ G. A. Holzapfel and R. W. Ogden, "Constitutive modelling of passive myocardium. A structurally-based framework for material characterization," Philos. Trans. R. Soc. London, Ser. A 367, 3445-3475 (2009).

${ }^{29}$ Y. Hu, V. Gurev, J. Constantino, J. D. Bayer, and N. A. Trayanova, "Effects of mechano-electric feedback on scroll wave stability in human ventricular fibrillation," PLoS One 8(4), e60287 (2013).

${ }^{30} \mathrm{H}$. Hu and F. Sachs, "Stretch-activated ion channels in the heart," J. Mol. Cell. Cardiol. 29, 1511-1523 (1997).

${ }^{31}$ J. Huang, J. M. Rogers, C. R. Killingswoth, K. P. Singh, W. M. Smith, and R. Ideker, "Evolution of activation patterns during long-duration ventricular fibrillation in dogs," Am. J. Physiol. Heart Circ. Physiol. 286, H1193-H1200 (2004). 
${ }^{32}$ P. J. Hunter, A. D. McCulloch, and H. E. D. J. ter Keurs, "Modelling the mechanical properties of cardiac muscle," Prog. Biophys. Mol. Biol. 69, 289-331 (1998).

${ }^{33}$ X. Jie, V. Gurev, and N. A. Trayanova, "Mechanisms of mechanically induced spontaneous arrhythmias in acute regional ischemia," Circ. Res. 106, 185-192 (2010).

${ }^{34}$ A. Kamkin, I. Kiseleva, K. D. Wagner, K. P. Leiterer, H. Theres, H. Scholz, J. Gunther, and M. J. Lab, "Mechano-electric feedback in right atrium after left ventricular infarction in rats," J. Mol. Cell. Cardiol. 32, 465-477 (2000).

${ }^{35}$ R. H. Keldermann, M. P. Nash, H. Gelderblom, V. Y. Wang, and A. V. Panfilov, "Electromechanical wavebreak in a model of the human left ventricle," Am. J. Physiol. Heart Circ. Physiol. 299, H134-H143 (2010).

${ }^{36}$ R. C. P. Kerckhoffs, P. H. M. Bovendeerd, J. C. S. Kotte, F. W. Prinzen, K. Smits, and T. Arts, "Homogeneity of cardiac contraction despite physiological asynchrony of depolarization: A model study," Ann. Biomed. Eng. 31, 536-547 (2003).

${ }^{37}$ R. C. P. Kerckhoffs, O. P. Faris, P. H. M. Bovendeerd, F. W. Prinzen, K. Smits, E. R. McVeigh, and T. Arts, "Electromechanics of paced left ventricle simulated by straightforward mathematical model: Comparison with experiments," Am. J. Physiol. Heart Circ. Physiol. 289, H1889-H1897 (2005).

${ }^{38}$ P. Kohl, F. Sachs, and M. R. Franz, Cardiac Mechano-Electric Coupling and Arrhythmias, 2th ed. (Oxford University Press, Oxford, 2011).

${ }^{39} \mathrm{P}$. Kohl and F. Sachs, "Mechanoelectric feedback in cardiac cells," Philos. Trans. R. Soc. London, Ser. A. 359, 1173-1185 (2001).

${ }^{40} \mathrm{P}$. Kohl, P. Hunter, and D. Noble, "Stretch-induced changes in heart rate and rhythm: Clinical observations, experiments and mathematical models," Prog. Biophys. Mol. Biol. 71, 91-138 (1999).

${ }^{41}$ S. Land, S. A. Niederer, J. M. Aronsen, E. K. S. Espe, L. L. Zhang, W. E. Louch, I. Sjaastad, O. M. Sejersted, and N. P. Smith, "An analysis of deformation-dependent electromechanical coupling in the mouse heart," J. Physiol. 590, 4553-4569 (2012).

${ }^{42}$ S. Land, S. A. Niederer, and N. P. Smith, "Efficient computational methods for solving strongly coupled cardiac electromechanics," IEEE Trans. Biomed. Eng. 59(5), 1219-1228 (2012).

${ }^{43}$ J. I. Laughner, S. Zhang, H. Li, C. C. Shao, and I. R. Efimov, "Mapping cardiac surface mechanics with structured light imaging," Am. J. Physiol. Heart Circ. Physiol. 303, H712-H720 (2012).

${ }^{44}$ W. Li, V. Gurev, A. D. McCulloch, and N. A. Trayanova, "The role of mechanoelectric feedback in vulnerability to electric shock," Prog. Biophys. Mol. Biol. 97, 461-478 (2008).

${ }^{45}$ X. T. Li, V. Dyachenko, M. Zuzarte, C. Putzke, R. Preisig-Muller, G. Isenberg, and J. Daut, "The stretch-activated potassium channel TREK-1 in rat cardiac ventricular muscle," Cardiovasc. Res. 69, 86-97 (2006).

${ }^{46} \mathrm{~W}$. Li, P. Kohl, and N. Trayanova, "Myocardial ischemia lowers precordial thump efficacy: An inquiry into mechanisms using three-dimensional simulations," Heart Rhythm 3, 179-186 (2006).

${ }^{47}$ M. P. Nash, A. Mourad, R. H. Clayton, P. M. Sutton, C. P. Bradley, M. Hayward, D. J. Peterson, and P. Taggart, "Evidence for multiple mechanisms in human ventricular fibrillation," Circulation 114, 536-542 (2006).

${ }^{48}$ M. P. Nash and A. V. Panfilov, "Electromechanical model of excitable tissue to study reentrant cardiac arrhythmias," Prog. Biophys. Mol. Biol. 85, 501-522 (2004).

${ }^{49}$ S. A. Niederer, P. J. Hunter, and N. P. Smith, "A quantitative analysis of cardiac myocyte relaxation: a simulation study," Biophys. J. 90, 1697-1722 (2006).

${ }^{50}$ S. A. Niederer and N. P. Smith, "A mathematical model of the slow force response to stretch in rat ventricular myocites," Biophys. J. 92, 4030-4044 (2007).

${ }^{51}$ S. A. Niederer and N. P. Smith, "An improved numerical method for strong coupling of excitation and contraction models in the heart," Prog. Biophys. Mol. Biol. 96, 90-111 (2008).

${ }^{52}$ P. J. Pathmanathan and J. P. Whiteley, "A numerical method for cardiac mechanoelectric simulations," Ann. Biomed. Eng. 37, 860-873 (2009).

${ }^{53}$ L. F. Pavarino and S. Scacchi, "Multilevel additive Schwarz preconditioners for the Bidomain reaction-diffusion system," SIAM J. Sci. Comput. 31, 420-443 (2008).
${ }^{54}$ L. F. Pavarino, S. Scacchi, and S. Zampini, "Newton-Krylov-BDDC solvers for non-linear cardiac mechanics," Comput. Methods Appl. Mech. Eng. 295, 562-580 (2015).

${ }^{55}$ R. Peyronnet, J. M. Nerbonne, and P. Kohl, "Cardiac mechano-gated ion channels and arrhythmias," Circ. Res. 118, 311-329 (2016).

${ }^{56}$ Z. Qu, J. Kil, F. Xie, A. Garfinkel, and J. N. Weiss, "Scroll wave dynamics in a three-dimensional cardiac tissue model: Roles of restitution, thickness, and fiber rotation," Biophys. J. 78, 2761-2775 (2000).

${ }^{57}$ Z. Qu, G. Hu, A. Garfinkel, and J. N. Weiss, "Nonlinear and stochastic dynamics in the heart," Phys. Rep. 534, 61-162 (2014).

${ }^{58}$ J. J. Rice, F. Wang, D. M. Bers, and P. P. de Tombe, "Approximate model of cooperative activation and cross bridge cycling in cardiac muscle using ordinary differential equations," Biophys. J. 95, 2368-2390 (2008).

${ }^{59}$ G. K. Rohde, B. M. Dawant, and L. Shien-Fong, "Correction of motion artifact in cardiac optical mapping using image registration," IEEE Trans. Biomed. Eng. 52, 338-341 (2005).

${ }^{60}$ S. Rossi, R. Ruiz-Baier, L. F. Pavarino, and A. Quarteroni, "Orthotropic active strain models for the numerical simulation of cardiac biomechanics," Int. J. Numer. Methods Biomed. Eng. 28, 761-788 (2012).

${ }^{61}$ J. Sainte-Marie, D. Chapelle, R. Cimrman, and M. Sorine, "Modeling and estimation of cardiac electromechanical activity," Comp. Struct. 84, 1743-1759 (2006).

${ }^{62}$ K. Seo, M. Inagaki, S. Nishimura, I. Hidaka, M. Sugimachi, T. Hisada, and S. Suguira, "Structural heterogeneity in ventricular wall plays a significant in the initiation of stretch-induced arrhythmias in perfused rabbit right ventricular tissues and whole heart preparations," Circ. Res. 106, 176-184 (2010).

${ }^{63}$ K. H. W. J. ten Tusscher, D. Noble, P. J. Noble, and A. V. Panfilov, "A model for human ventricular tissue,” Am. J. Phys. Heart Circ. Physiol. 286, H1573-H1589 (2004).

${ }^{64}$ K. H. W. J. ten Tusscher and A. V. Panfilov, "Alternans and spiral breakup in a human ventricular tissue model," Am. J. Phys. Heart Circ. Physiol. 291, H1088-H1100 (2006).

${ }^{65} \mathrm{~K}$. H. W. J. ten Tusscher and A. V. Panfilov, "Organization of ventricular fibrillation in the human heart," Circ. Res. 100, e87-e101 (2007).

${ }^{66} \mathrm{~N}$. Trayanova, W. Li, J. Eason, and P. Kohl, "Effect of stretch-activated channels on defibrillation efficacy," Heart Rhythm 1, 67-77 (2004).

${ }^{67}$ T. P. Usyk, I. J. LeGrice, and A. D. McCulloch, "Computational model of three-dimensional cardiac electromechanics," Comput. Visualization Sci. 4, 249-257 (2002).

${ }^{68}$ T. P. Usyk and A. D. McCulloch, "Electromechanical model of cardiac resychronization in the dilated failing heart with left bundle branch block," J. Electrocardiol. 36, 57-61 (2003).

${ }^{69}$ C. Vergara, M. Lange, S. Palamara, T. Lassila, A. F. Frangi, and A. Quarteroni, "A coupled 3D - 1D numerical monodomain solver for cardiac electrical activation in the myocardium with detailed Purkinje network," J. Comput. Phys. 308, 218-238 (2016).

${ }^{70}$ F. J. Vetter and A. D. McCulloch, "Mechanoelectric feedback in a model of the passively inflated left ventricle," Ann. Biomed. Eng. 29, 414-426 (2001).

${ }^{71}$ F. Xie, Z. Qu, J. Yang, A. Baher, J. Weiss, and A. Garfinkel, "A simulation study of the effects of cardiac anatomy in ventricular fibrillation," J. Clin. Invest. 113, 686-693 (2004).

${ }^{72}$ M. Zabel, B. S. Koller, F. Sachs, and M. R. Franz, "Stretch-induced voltage changes in the isolated beating heart: Importance of the timing of stretch and implications for stretch-activated ion channels," Cardiovasc. Res. 32, 120-130 (1996).

${ }^{73}$ H. Zhang, K. Iijima, J. Huang, G. P. Walcott, and J. M. Rogers, “Optical mapping of membrane potential and epicardial deformation in beating hearts," Biophys. J. 111, 438-451 (2016).

${ }^{74}$ T. Zeng, G. C. L. Bett, and F. Sachs, "Stretch-activated whole cell currents in adult rat cardiac myocytes," Am. J. Physiol. Heart Circ. Physiol. 278, H548-H557 (2000).

${ }^{75}$ S. T. Wall, J. M. Guccione, M. B. Ratcliffe, and J. S. Sundnes, "Electromechanical feedback with reduced cellular connectivity alters electrical activity in an infarct injures left ventricle: A finite element model study," Am J. Physiol. Heart Circ. Physiol. 302, H206-H214 (2012).

${ }^{76}$ J. N. Weiss, Z. Qu, P. S. Chen, S. F. Lin, H. S. Karagueuzian, H. Hayashi, A. Garfinkel, and A. Karma, "The dynamics of cardiac fibrillation," Circulation 112, 1232-1240 (2005). 